INTERNATIONAL JOURNAL
PHARMACEUTICAL SCIENCES
RESEARCH
RESTI

Received on 17 May 2019; received in revised form, 14 October 2019; accepted, 29 January 2020; published 01 March 2020

\title{
DESIGN, SYNTHESIS AND BIOLOGICAL EVALUATION OF NOVEL QUINOLINE ANALOGUES AS HIV-1 INTEGRASE INHIBITOR
}

\author{
K. D. Deo ${ }^{1}$, I. J. Singhvi ${ }^{2}$, S. Murugesan ${ }^{3}$, G. P. Vadnere ${ }^{4}$ and A. V. Patil ${ }^{*} 4$
}

Faculty of Pharmacy 1,2, Pacific Academy of Higher Education and Research University, Udaipur 313001, Rajasthan, India.

Medicinal Chemistry Research Laboratory ${ }^{3}$, Department of Pharmacy, Birla Institute of Technology and Science, Pilani Campus, Pilani - 333031, Rajasthan, India.

Smt. S. S. Patil College of Pharmacy ${ }^{4}$, Chopda, Jalgaon - 425107, Maharashtra, India.

Keywords:

Quinoline, Elvitegravir, Docking, HIV-1 integrase, Raltegravir, Syncytium

\section{Correspondence to Author:}

Prof. Dr. Avinash V. Patil

Smt. S. S. Patil College

of Pharmacy, Chopda, Jalgaon -

425107, Maharashtra, India.

E-mail: avinashay_princ@rediffmail.com
ABSTRACT: A progression of fourteen narrative quinolonyl diketo acid analogs were planned, synthesized, identified by IR, NMR, CHN and MS supernatural analysis and evaluated as potential HIV-1 Integrase hinders. Compounds of Zinc database were surfed considering Elvitegravir as standard. Nearly 99 compounds were identified and docked in the active site of HIV-1 integrase. Molecular docking study of compounds 1, 4 and 7 showed docking score $-10.38,-9.31$ and -10.12 respectively as that of set drug Elvitegravir -4.93 . The docking poses to open the interaction of the ligands with preferred amino acids. The standard drug Elvitegravir displayed connections with lys156, Asn155, Lys159 and Thr66. Raltegravir showed hydrogen bonding with Asp 116. A round fourteen target diketoquinolines were chosen for advance synthesis with the help of substituted oxoquinoline-3-carboxylate as starting material. Invitro biological evaluation open that some of the upper-class compounds exhibited moderate to good inhibitory activity besides HIV1 Integrase compared to the reference drugs Raltegravir and Nevirapine. Compounds 1, 2, 3 and 4 weakly inhibited HIV-1 integrase at EC50 of $0.31,0.25,0.22 \& 0.21$ with the therapeutic index 242, 260, 266 and 278 respectively. The cytotoxicity of upperclass compounds on C8166 cells was very low, the CC50 value was higher than $200 \mu \mathrm{M}$, except for few compounds. As an optimistic control drug, Nevirapine showed significant anti-HIV-1 activity $\left(\mathrm{EC}_{50}=0.015 \sim 0.016 \mu \mathrm{M}\right)$ in-vitro, and the $\mathrm{CC}_{50}$ was higher than $200 \mu \mathrm{M}$, with a therapeutic index value of 12418.50 . Compound 14 exhibited significant inhibition of HIV-1 syncytium and integrase at $\mathrm{EC}_{50} 0.25$ and 0.12 respectively.
INTRODUCTION: Retrieving the first case of acquired immunodeficiency syndrome (AIDS) of 1981 the Disease Expert Group (DEG) recently put forth the fact about nearly 40 million individuals were infected by HIV/ AIDS.

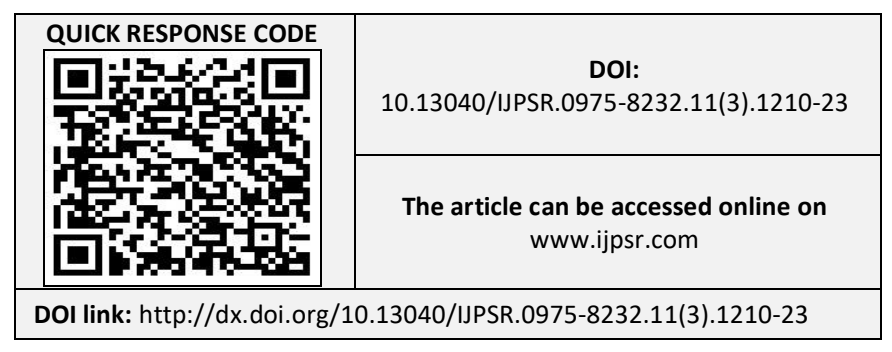

The extent of antiretroviral therapy posing the success to change the infection of HIV from a deadly disease to controllable chronic disease significantly for the last three decades. Thus, the endurance in life expectancy has surged amongst folk infected with HIV ${ }^{1,2}$.

Etiology and prevalence of the infection shown to have very rapid development of drug resistance to many existing drug classes and warrant for the discovery of new targets. Among the three major enzymes, i.e. HIV-1 protease, HIV-1 reverse transcriptase and HIV-1 integrase [IN]) of the viral 
replication cycle, HIV-1 IN has been of particular interest due to the absence of human cellular homolog. HIV-1 IN process the integration of viral genetic material with the host genome, a key step in the viral duplication process. Several novel classes of HIV IN inhibitors have been explored by targeting different sites on the enzyme ${ }^{3,4}$.

Persistent efforts were on to the development of the new HIV-1 duplication antiretroviral agents with significant efficacy equally should be devoid of inherent toxicity, development of drug resistance, and poor tolerability. The integrase enzyme is required to repeat HIV-1 catalyzes the integration of reverse-transcribed DNA into the genome of the host cell. Therefore, integration has emerged as an attractive site according to the review of the different targets. The structural study advocated that IN is the active site inhibited by the Raltegravir and Elvitegravir, needs a single binding site for $\mathrm{Mg}^{2+}$ metal ions. Several integrase inhibitors having metal-binding potential have been illustrated as well as published by several researchers ${ }^{5-9}$.

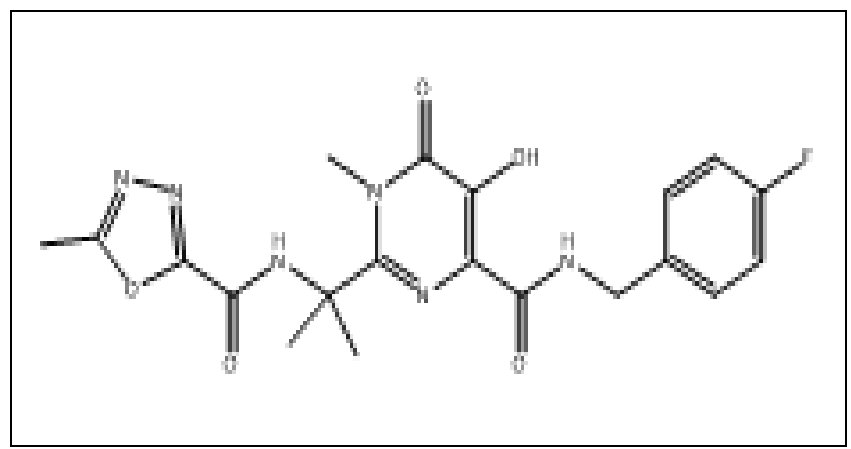

RALTEGRAVIR

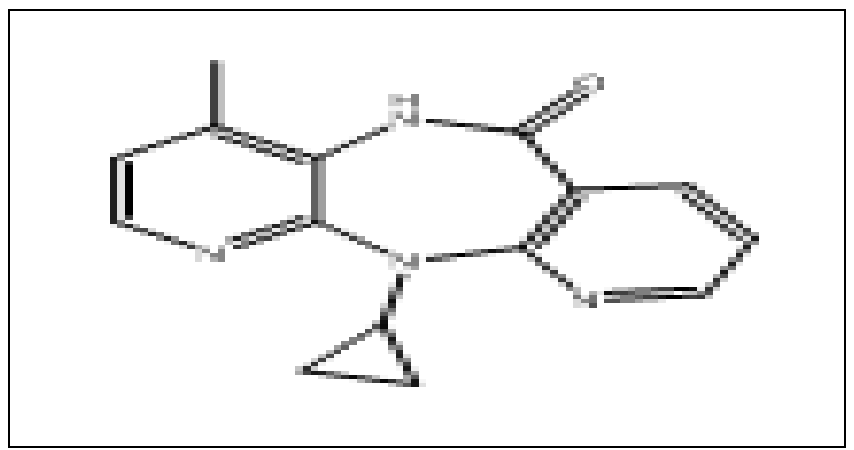

NEVIRAPINE

FIG. 1: REPRESENTATIVE OF HIV-1 INHIBITORS

The diketoquinolines were first designed and few active compounds were prepared using oxo quinoline-3-carboxylate compounds with substituted piperazine, benzoic acid, 2-phenoxy
As a continuation of a series of many reviews on synthetic approaches and final forms of recently approved drugs on the design and development of routes to the integrase inhibitors such as Dolutegravir, Cabotegravir, and Bictegravir was found predominantly ${ }^{10}$. Compounds like $\beta$-diketo acid (DKA) has emerged as the most powerful and promising blocker, Raltegravir is the first approved IN hindering to reach up to clinical development along with Elvitegravir and GSK364735 as shown in Fig. 1.

These DKA hindering share two common structural chemotypes essential for anti IN movement: a sequence of diketo acid which is capable to interact with $\mathrm{Mg}^{2+}$ metal ions and a properly oriented moiety of hydrophobic benzyl. Manipulative of identical analogs targeting to integrase may give rise to the newer ideal drug to cure AIDS and decrease the unwanted results of the previous compounds and may be responsible for new generation integrase blockers ${ }^{11-13}$.

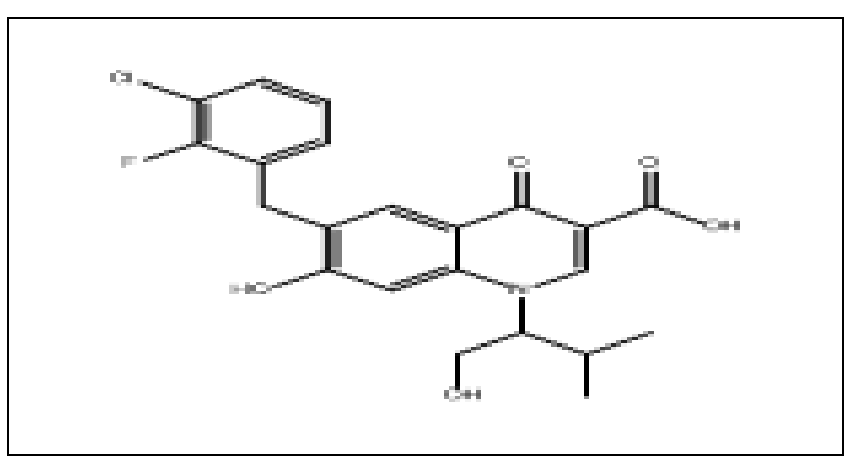

ELVITEGRAVIR

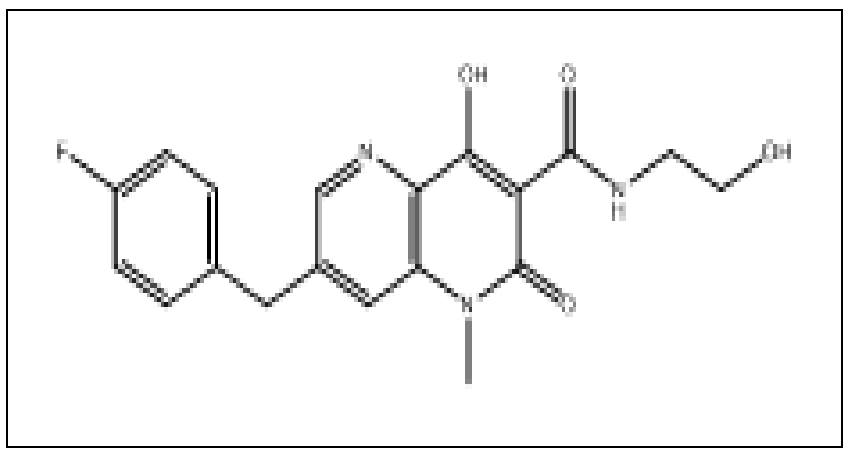

GSK364735

acetic acid and benzene-1-sulfonyl chloride. The study was performed with structure-based virtual screening approaches. In this context, we synthesized narrative quinolone derivatives 1-14 
and evaluated for their anti-HIV-1 integrase activity.

\section{MATERIALS AND METHODS: Chemistry:}

General: The reagents and solvents were used as usual. Reactions were observed by TLC with silica gel plates. Silica gel (100-200 mesh) as a fixed phase was used for column chromatography. The melting points were firm were not corrected. MS spectra of the synthesized analogs were documented on the Shimadzu QP-5050 spectrophotometer. 1H NMR spectra were acquired on a Varian-300 (300 MHz NMR) spectrophotometer using $\mathrm{CDCl}_{3}$ and DMSO-d6 as a solvent.

The infrared (IR) spectra were retrieved using Perkin Elmer Spectrum ES Version 10.5.3 Fouriertransform infrared spectrometer. Elemental analysis was performed on FLASH EA 1112 CHN Elemental analyzer, Thermofinnigen, Italy.

\section{General Procedure for Synthesis of Compounds (1-6):}

Methy1-Cyclopropyl-6-Fluoro-1, 4-Hydro-4Oxo-7-(Substituted Piperzin-1-Yl) Quinoline-3Carboxylate (1a-6a): Added methyl 7-chloro-1cyclopropyl-6-fluoro-1,4-dihydro-4-oxoquinoline-

3 -carboxylate ( $1 \mathrm{~g}, 3.53 \mathrm{mmole})$ in acetonitrile (10 $\mathrm{ml}$ ), shacked the reaction mixture at $35{ }^{\circ} \mathrm{C}$, added DIPEA (N, Ndi isopropyl ethylamine) $(0.5 \mathrm{ml})$ and substituted piperazine ( $3.53 \mathrm{~m}$ mole) to the reaction mixture, shacked at boiling temperature for $24 \mathrm{~h}$. The reaction mixture was shacked with Ethyl acetate, dried over sodium sulphate, concentrated under vacuum. The basic product was cleansed by column chromatography using silica gel (100-200 mesh) to get methy1-cyclopropyl-6-fluoro-1, 4hydro - 4 - oxo-7 - (substituted piperzin - 1-yl) quinoline-3-carboxylate as off-White solid.

Comp. 1a: Yield (0.9 g, 75\%), MP-198-200 ${ }^{\circ} \mathrm{C}$.

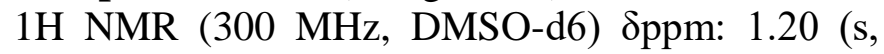
$1 \mathrm{H}$, cyclopropane $\mathrm{H}$ ), 1.32-1.36 (d, 4H, cyclo propane $\mathrm{CH}_{2}$ ), 3.32 (br-s, $4 \mathrm{H}$, piperazine $\mathrm{CH}_{2}$ ), 3.60 (br-s, 4H, piperazine $\mathrm{CH}_{2}$ ), $3.73(\mathrm{~s}, 3 \mathrm{H}$, $\left.\mathrm{OCH}_{3}\right), 3.80(\mathrm{~m}, 1 \mathrm{H}, \mathrm{NH}), 7.60-7.62(\mathrm{~d}, 1 \mathrm{H}, \mathrm{ArH})$, 7.90-7.95 (d, 1H, Ar H), 8.68 (s, 1H, N-C=C-H). IR $(\mathrm{KBr}) \mathrm{cm}^{-1}: 2925(\mathrm{C}-\mathrm{H} \mathrm{Ar}), 1710\left(\mathrm{C}=\mathrm{OOCH}_{3}\right)$, $1680(\mathrm{C}=\mathrm{O}$ quinoline $), 1562,1493(\mathrm{C}=\mathrm{C} \mathrm{Ar}), 1450$ (C-N st), 1384 (C-N-C=), 1260 (C-O-C).
Comp. 2a: Yield (0.85 g, 70\%), MP-162-164 ${ }^{\circ} \mathrm{C}$.

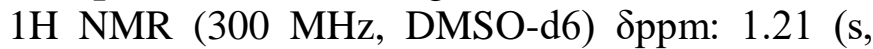
$1 \mathrm{H}$, cyclo propane $\mathrm{H}), 1.26(\mathrm{q}, 1 \mathrm{H}$, piperazine $\mathrm{H})$, 1.33-1.36 (d, 4H, cyclopropane $\left.\mathrm{CH}_{2}\right), 1.41$ (d, 3H, $\mathrm{CH}_{3}$ ), 3.10 (br-s, $2 \mathrm{H}$, piperazine $\mathrm{CH}_{2}$ ), 3.40 (br-s, $4 \mathrm{H}$, piperazine $\left.\mathrm{CH}_{2}\right), 3.72\left(\mathrm{~s}, 3 \mathrm{H}, \mathrm{OCH}_{3}\right), 3.85$ (m, $1 \mathrm{H}, \mathrm{NH}), 7.56-7.62(\mathrm{~d}, 1 \mathrm{H}, \mathrm{Ar} H), 7.88-7.95(\mathrm{~d}$, $1 \mathrm{H}, \mathrm{Ar} \mathrm{H}), 8.66$ (s, $1 \mathrm{H}, \mathrm{N}-\mathrm{C}=\mathrm{C}-\mathrm{H})$. IR $(\mathrm{KBr}) \mathrm{cm}^{-1}$ : 2935 (C-H Ar), $1712\left(\mathrm{C}=\mathrm{OOCH}_{3}\right), 1680(\mathrm{C}=\mathrm{O}$ quinoline), 1564, 1492 (C=C Ar), 1455 (C-N st), 1382 (C-N-C=), 1260 (C-O-C).

Comp. 3a: Yield $(0.80 \mathrm{~g}, 63 \%)$, MP-204-206 ${ }^{\circ} \mathrm{C}$.

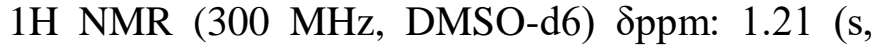
$1 \mathrm{H}$, cyclopropane $\mathrm{H}), 1.25$ (q, $2 \mathrm{H}$, piperazine $\mathrm{H}$ ), 1.33-1.36 (d, 4H, cyclopropane $\left.\mathrm{CH}_{2}\right), 1.41(\mathrm{~d}, 6 \mathrm{H}$, $\left.2 \mathrm{CH}_{3}\right), 2.90$ (br-s, 4H, piperazine $\left.\mathrm{CH}_{2}\right), 3.70(\mathrm{~s}$, $\left.3 \mathrm{H}, \mathrm{OCH}_{3}\right), 3.82(\mathrm{~m}, 1 \mathrm{H}, \mathrm{NH}), 7.50-7.62(\mathrm{~d}, 1 \mathrm{H}$, $\operatorname{Ar} \mathrm{H}), 7.88-7.90(\mathrm{~d}, 1 \mathrm{H}, \operatorname{Ar} \mathrm{H}), 8.64(\mathrm{~s}, 1 \mathrm{H}, \mathrm{N}-$ $\mathrm{C}=\mathrm{C}-\mathrm{H})$. IR $(\mathrm{KBr}) \mathrm{cm}^{-1}$ : 2939 (C-H Ar), 1710 $\left(\mathrm{C}=\mathrm{OOCH}_{3}\right), 1678(\mathrm{C}=\mathrm{O}$ quinoline $), 1565,1490$ $(\mathrm{C}=\mathrm{C} \mathrm{Ar}), 1453$ (C-N st), $1380(\mathrm{C}-\mathrm{N}-\mathrm{C}=), 1260$ (C$\mathrm{O}-\mathrm{C})$.

Comp. 4a: Yield (0.95 g, 78\%), MP-170-172 ${ }^{\circ} \mathrm{C}$.

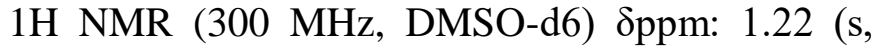
$1 \mathrm{H}$, cyclopropane $\mathrm{H}), \quad 1.32-1.38 \quad(\mathrm{~d}, 4 \mathrm{H}$, cyclopropane $\mathrm{CH} 2$ ), 3.22 (s, $3 \mathrm{H}, \mathrm{N}-\mathrm{CH}_{3}$ ), 3.34 (br$\mathrm{s}, 4 \mathrm{H}$, piperazine $\left.\mathrm{CH}_{2}\right), 3.61$ (br-s, $4 \mathrm{H}$, piperazine $\mathrm{CH} 2), 3.72$ (s, 3H, $\left.\mathrm{OCH}_{3}\right)$, 7.60-7.64 (d, 1H, Ar H), 7.92-7.98 (d, 1H, Ar H), 8.66 (s, 1H, N-C=C-H). IR $(\mathrm{KBr}) \mathrm{cm}^{-1}: 2941$ (C-H Ar), $1714\left(\mathrm{C}=\mathrm{OOCH}_{3}\right)$, $1678(\mathrm{C}=\mathrm{O}$ quinoline $), 1566,1492(\mathrm{C}=\mathrm{C}$ Ar $), 1460$ (C-N st), 1382 (C-N-C=), 1260 (C-O-C).

Comp. 5a: Yield $(0.90 \mathrm{~g}, 70 \%)$, MP-168-170 ${ }^{\circ} \mathrm{C}$.

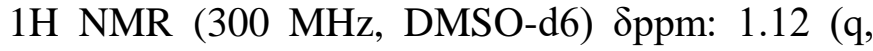
$1 \mathrm{H}$, piperazine $\mathrm{H}), 1.16-1.25\left(\mathrm{t}, 3 \mathrm{H}, \mathrm{CH}_{3}\right), 1.21$ (s, $1 \mathrm{H}$, cyclopropane $\mathrm{H}), \quad 1.33-1.36(\mathrm{~d}, \quad 4 \mathrm{H}$, cyclopropane $\left.\mathrm{CH}_{2}\right), 1.40\left(\mathrm{~d}, 3 \mathrm{H}, \mathrm{NCH}_{2} \mathrm{CH}_{3}\right), 3.10$ (br-s, 4H, piperazine $\mathrm{CH}_{2}$ ), 3.40 (br-s, 4H, piperazine $\mathrm{CH} 2), 3.74\left(\mathrm{~s}, 3 \mathrm{H}, \mathrm{OCH}_{3}\right), 3.91(\mathrm{q}, 2 \mathrm{H}$, $\mathrm{NCH}_{2} \mathrm{CH}_{3}$ ), 7.57-7.62 (d, 1H, Ar H), 7.88-7.97 (d, $1 \mathrm{H}, \mathrm{Ar} \mathrm{H}), 8.68$ (s, 1H, N-C=C-H). IR $(\mathrm{KBr}) \mathrm{cm}^{-1}$ : 2930 (C-H Ar), $1710\left(\mathrm{C}=\mathrm{OOCH}_{3}\right), 1680(\mathrm{C}=\mathrm{O}$ quinoline), 1564, 1492 (C=C Ar), 1459 (C-N st), $1382(\mathrm{C}-\mathrm{N}-\mathrm{C}=), 1260$ (C-O-C).

Comp. 6a: Yield (0.95 g, 70\%), MP- 182-184 ${ }^{\circ} \mathrm{C}$.

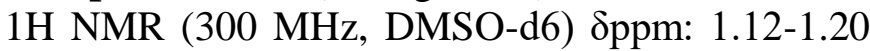
(t, $\left.3 \mathrm{H}, \mathrm{NCH}_{2} \mathrm{CH}_{3}\right), 1.25$ (q, $2 \mathrm{H}$, piperazine $\left.\mathrm{H}\right), 1.30$ 
(s, 1H, cyclopropane H), 1.33-1.38 (d, 4H, cyclopropane $\mathrm{CH} 2$ ), 3.92 (q, $2 \mathrm{H}, \mathrm{NCH}_{2} \mathrm{CH}_{3}$ ), 1.45 (d, 6H, 2 $\mathrm{CH}_{3}$ ), 2.90 (br-s, $4 \mathrm{H}$, piperazine $\mathrm{CH}_{2}$ ), $3.78\left(\mathrm{~s}, 3 \mathrm{H}, \mathrm{OCH}_{3}\right), 7.50-7.62(\mathrm{~d}, 1 \mathrm{H}, \mathrm{Ar} \mathrm{H}), 7.88-$ $7.90(\mathrm{~d}, 1 \mathrm{H}, \operatorname{Ar} \mathrm{H}), 8.62(\mathrm{~s}, 1 \mathrm{H}, \mathrm{N}-\mathrm{C}=\mathrm{C}-\mathrm{H})$. IR $(\mathrm{KBr}) \mathrm{cm}^{-1}: 2932$ (C-H Ar), $1710\left(\mathrm{C}=\mathrm{OOCH}_{3}\right)$, $1681(\mathrm{C}=\mathrm{O}$ quinoline), 1565, $1490(\mathrm{C}=\mathrm{C} \mathrm{Ar}), 1456$ (C-N st), 1380 (C-N-C=), 1260 (C-O-C).

1-Cyclopropyl-6-Fluoro-1，4-Dihydro-4-Oxo-7(Substituted Piperazin-1-Yl) Quinoline-3Carboxylic Acid (1-6): A solution of methy1cyclopropyl-6-fluoro-1,4-hydro-4-oxo-7 (substituted piperzin-1-yl) quinoline-3- carboxylate $(2.60 \mathrm{~m}$ mole) in Methanol $(12.6 \mathrm{ml})$ and water $(5.4 \mathrm{ml})$, LiOH. $\mathrm{H}_{2} \mathrm{O}(0.34 \mathrm{ml}, 8.10 \mathrm{~m} \mathrm{~mol})$ was added at room temperature. The reaction mixture was shacked at room temperature for $6 \mathrm{~h}$. until the TLC showed the completion of starting matter. The basic product was refined under vacuum, neutralized with $1 \mathrm{~N} \mathrm{HCl}$ infused with dichloromethane, separated organic layer was dried over sodium sulphate, intensed under vacuum and washed with hexane to get 1-cyclopropyl-6-fluoro-1, 4-dihydro4-oxo-7-(substituted piperazin-1-yl) quinoline-3carboxylic acid as white solid.

Comp. 1: Yield (73\%, 0.62 g). MP - 161-163 ${ }^{\circ} \mathrm{C}$.

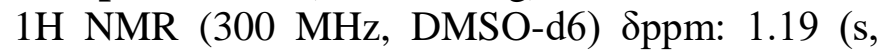
$1 \mathrm{H}$, cyclopropane $\mathrm{H}), \quad 1.32-1.34 \quad(\mathrm{~d}, 4 \mathrm{H}$, cyclopropane $\mathrm{CH}_{2}$ ), 3.31 (br-s, $4 \mathrm{H}$, piperazine $\left.\mathrm{CH}_{2}\right), 3.57$ (br-s, $4 \mathrm{H}$, piperazine $\left.\mathrm{CH}_{2}\right), 3.86(\mathrm{~m}, 1 \mathrm{H}$, $\mathrm{NH})$, 7.59-7.61 (d, 1H, Ar H), 7.90-7.95 (d, 1H, Ar $\mathrm{H}), 8.66$ (s, 1H, N-C=C-H), 9.57 (s, 1H, COOH).IR $(\mathrm{KBr}) \mathrm{cm}^{-1}: 3510(\mathrm{COOH}), 2924(\mathrm{C}-\mathrm{H} \mathrm{Ar}), 1706$ $(\mathrm{C}=\mathrm{O}, \mathrm{COOH}), 1680(\mathrm{C}=\mathrm{O}$ quinoline $), 1562,1493$ (C=C Ar), 1448 (C-N st), 1383 (C-N-C=). MS: m/z (relint \%) 331 (32) [M]+, 255 (27), 253 (15), 219 (100), 205 (34), 199 (65), 183(18), 182 (45), 156 (32), 144 (20), 113 (80). CHN Analysis: $\mathrm{C}_{17} \mathrm{H}_{18} \mathrm{FN}_{3} \mathrm{O}_{3} \mathrm{Cal}: \mathrm{C} 61.56 \%$, H 5.43\%, N 12.67\%, Found: C 61.59\%, H 5.45\%, N 12.68\%,

Comp. 2: Yield $\left(81 \%, 0.70\right.$ g). MP - $148-150{ }^{\circ} \mathrm{C}$.

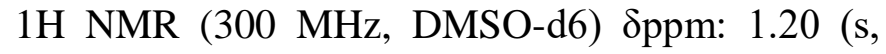
$1 \mathrm{H}$, cyclopropane $\mathrm{H}$ ), 1.25 (q, $1 \mathrm{H}$, piperazine $\mathrm{CH}$ ), 1.34-1.36 (d, 4H, cyclopropane $\left.\mathrm{CH}_{2}\right), 1.42(\mathrm{~d}, 3 \mathrm{H}$, $\mathrm{CH}_{3}$ ), 3.22 (br-s, $2 \mathrm{H}$, piperazine $\mathrm{CH}_{2}$ ), 3.58 (br-s, $4 \mathrm{H}$, piperazine $\left.\mathrm{CH}_{2}\right), 3.88(\mathrm{~m}, 1 \mathrm{H}, \mathrm{NH}), 7.59-7.62$ (d, 1H, Ar H), 7.90-7.95 (d, 1H, Ar H), 8.66 (s, 1H, $\mathrm{N}-\mathrm{C}=\mathrm{C}-\mathrm{H}), 9.60(\mathrm{~s}, 1 \mathrm{H}, \mathrm{COOH})$. IR $(\mathrm{KBr}) \mathrm{cm}^{-1}$ :
$3550(\mathrm{COOH}), 2926(\mathrm{C}-\mathrm{H}$ Ar $), 1705(\mathrm{C}=\mathrm{O}$, $\mathrm{COOH}), 1680(\mathrm{C}=\mathrm{O}$ quinoline $), 1562,1494(\mathrm{C}=\mathrm{C}$ Ar), 1446 (C-N st), 1382 (C-N-C=). MS: m/z (relint \%) 345 (38) [M] +, 330 (45), 255 (66), 253 (37), 219 (100), 205 (68), 190 (73), 183 (53), 182 (51), 158 (48), 144 (65), 113 (68), 99 (70). CHN Analysis: $\mathrm{C}_{18} \mathrm{H}_{20} \mathrm{FN}_{3} \mathrm{O}_{3}$ Cal: $\mathrm{C}$ 62.60\%, H 5.84\%, N $12.17 \%$, Found: C 62.62\%, H 5.86\%, N $12.18 \%$.

Comp. 3: Yield $\left(80 \%, 0.60\right.$ g). MP- $172-174{ }^{\circ} \mathrm{C}$.

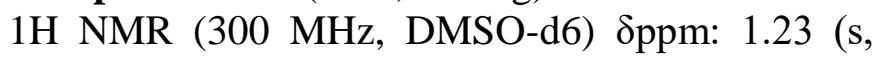
$1 \mathrm{H}$, cyclopropane $\mathrm{H}), 1.28$ (q, 2H, piperazine $\mathrm{C}-\mathrm{H})$, 1.32-1.36 (d, 4H, cyclopropane $\left.\mathrm{CH}_{2}\right), 1.42(\mathrm{~d}, 6 \mathrm{H}$, $2 \mathrm{CH}_{3}$ ), 3.20 (br-s, 4H, piperazine $\mathrm{CH}_{2}$ ), 3.84 (m, $1 \mathrm{H}, \mathrm{NH}), 7.60-7.62$ (d, 1H, Ar H), 7.90-7.95 (d, $1 \mathrm{H}, \operatorname{Ar} \mathrm{H}), 8.66(\mathrm{~s}, 1 \mathrm{H}, \mathrm{N}-\mathrm{C}=\mathrm{C}-\mathrm{H}), 9.60(\mathrm{~s}, 1 \mathrm{H}$, $\mathrm{COOH})$. IR (KBr) cm $\mathrm{cm}^{-1}: 3550(\mathrm{COOH}), 2928(\mathrm{C}-\mathrm{H}$ Ar), $1700(\mathrm{C}=\mathrm{O}, \mathrm{COOH}), 1680(\mathrm{C}=\mathrm{O}$ quinoline $)$, 1562, 1494 (C=C Ar), 1446 (C-N st), 1382 (C-N$\mathrm{C}=$ ). MS: $\mathrm{m} / \mathrm{z}$ (relint \%) 359 (22) [M] +, 340 (18), 315 (20), 290 (25), 274 (33), 255 (40), 253 (38), 219 (100), 199 (66), 205 (64), 183 (72), 182 (69), 156 (56), 144 (75), 113 (78). CHN Analysis: $\mathrm{C}_{19} \mathrm{H}_{22} \mathrm{FN}_{3} \mathrm{O}_{3} \mathrm{Cal}: \mathrm{C} 63.50 \%$, H 6.17\%, N 11.69\%, Found: C 63.52\%, H 6.74\%, N $11.70 \%$.

Comp. 4: Yield $(72 \%, 0.60 \mathrm{~g})$. MP $-152-154{ }^{\circ} \mathrm{C}$.

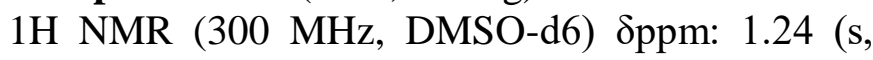
$1 \mathrm{H}$, cyclopropane $\mathrm{H}), 1.32-1.38$ (d, $4 \mathrm{H}$, cyclopropane $\mathrm{CH}_{2}$ ), 3.35 (br-s, $4 \mathrm{H}$, piperazine $\mathrm{CH}_{2}$ ), 3.50 (br-s, 4H, piperazine $\mathrm{CH}_{2}$ ), 3.83 (s, $3 \mathrm{H}, \mathrm{CH}_{3}$ ), 7.60-7.66 (d, 1H, Ar H), 7.90-7.95 (d, 1H, Ar H), $8.66(\mathrm{~s}, 1 \mathrm{H}, \mathrm{N}-\mathrm{C}=\mathrm{C}-\mathrm{H}), 9.60(\mathrm{~s}, 1 \mathrm{H}, \mathrm{COOH})$. IR $(\mathrm{KBr}) \mathrm{cm}^{-1}: 3550(\mathrm{COOH}), 2928(\mathrm{C}-\mathrm{H} \mathrm{Ar}), 1700$ $(\mathrm{C}=\mathrm{O}, \mathrm{COOH}), 1682(\mathrm{C}=\mathrm{O}$ quinoline $), 1562,1494$ $(\mathrm{C}=\mathrm{C}$ Ar), $1450(\mathrm{C}-\mathrm{N} \mathrm{st}), 1382(\mathrm{C}-\mathrm{N}-\mathrm{C}=), \mathrm{MS}: \mathrm{m} / \mathrm{z}$ (relint \%) 345 (28) [M+], 317 (22), 298 (26), 272 (31), 256 (48), 254 (47), 219 (100), 199 (57), 205 (60), 186 (72), 182 (69), 158 (72), 144 (34), 112 (75). CHN Analysis: $\mathrm{C}_{18} \mathrm{H}_{20} \mathrm{FN}_{3} \mathrm{O}_{3}$ Cal: $\mathrm{C}$ 62.60\%, $\mathrm{H} 5.84 \%$, N 12.17\%, Found: C 62.59\%, H 5.86\%, $\mathrm{N} 12.18 \%$.

Comp. 5: Yield (78\%, 0.67 g). MP- $156-158{ }^{\circ} \mathrm{C}$.

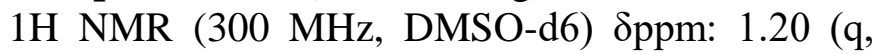
$\left.1 \mathrm{H}, \mathrm{HCCH}_{3}\right)$. 1.25-1.39 (t, 3H, $\left.\mathrm{CH}_{3}\right), 1.28(\mathrm{~s}, 1 \mathrm{H}$, cyclopropane $\mathrm{H}), 1.31-1.35$ (d, 4H, cyclopropane $\mathrm{CH} 2), 1.40\left(\mathrm{~d}, 3 \mathrm{H}, \mathrm{CH}_{3}\right), 3.30$ (br-s, $2 \mathrm{H}$, piperazine $\mathrm{CH} 2$ ), 3.50 (br-s, $4 \mathrm{H}$, piperazine $\mathrm{CH}_{2}$ ), 3.95 (q, $2 \mathrm{H}$, $\mathrm{CH} 2)$, 7.60-7.66 (d, 1H, Ar H), 7.90-7.95 (d, 1H, Ar H), $8.68(\mathrm{~s}, 1 \mathrm{H}, \mathrm{N}-\mathrm{C}=\mathrm{C}-\mathrm{H}), 9.60(\mathrm{~s}, 1 \mathrm{H}$, 
COOH). IR (KBr) cm ${ }^{-1}: 3550(\mathrm{COOH}), 2928(\mathrm{C}-\mathrm{H}$ Ar), $1700(\mathrm{C}=\mathrm{O}, \mathrm{COOH}), 1678(\mathrm{C}=\mathrm{O}$ quinoline $)$, 1566, 1494 (C=C Ar), 1450 (C-N st), 1382 (C-N$\mathrm{C}=$ ). MS: $\mathrm{m} / \mathrm{z}$ (relint \%) 373 (41) [M+], 352 (29), 320 (20), 292 (33), 277 (27), 244 (44), 240 (17), 222 (100), 199 (48), 190 (65), 183 (61), 182 (60), 158 (68), 144 (71), 131 (60), 112 (45). CHN Analysis: $\mathrm{C}_{20} \mathrm{H}_{24} \mathrm{FN}_{3} \mathrm{O}_{3}$ Cal: $\mathrm{C} 64.33 \%$, H 6.48\%, N 11.25\%, Found: C 64.32\%, H 6.50\%, N $11.26 \%$.

Comp. 6: Yield $\left(72 \%, 0.61\right.$ g). MP- $174-176{ }^{\circ} \mathrm{C}$.

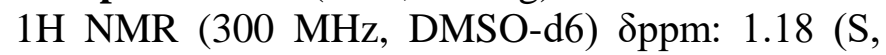
$1 \mathrm{H}$, cyclopropane $\mathrm{H}), 1.21$ (q, $\left.2 \mathrm{H}, \mathrm{HCCH}_{3}\right), 1.27$ $1.30\left(\mathrm{t}, 3 \mathrm{H}, \mathrm{CH}_{3}\right), 1.30-1.35$ (d, $4 \mathrm{H}$, cyclopropane $\left.\mathrm{CH}_{2}\right), \quad 1.41\left(\mathrm{~d}, 6 \mathrm{H}, 2 \mathrm{CH}_{3}\right), 3.28$ (br-s, 4H, piperazine $\left.2 \mathrm{CH}_{2}\right), 3.96\left(\mathrm{q}, 2 \mathrm{H}, \mathrm{CH}_{2}\right), 7.60-7.66(\mathrm{~d}$, 1H, Ar H), 7.90-7.95 (d, 1H, Ar H), 8.68 (s, 1H, N$\mathrm{C}=\mathrm{C}-\mathrm{H}), 9.60$ (s, 1H, COOH). IR $(\mathrm{KBr}) \mathrm{cm}^{-1}: 3550$ $(\mathrm{COOH}), 2930(\mathrm{C}-\mathrm{H} \mathrm{Ar}), 1700(\mathrm{C}=\mathrm{O}, \mathrm{COOH})$, 1680 (C=O quinoline), 1566, $1494(\mathrm{C}=\mathrm{C}$ Ar $), 1450$ (C-N st), $1382(\mathrm{C}-\mathrm{N}-\mathrm{C}=)$. MS: m/z (relint \%) 387 (21) [M+], 355 (18), 322 (18), 308 (16), 292 (21), 270 (34), 255 (33), 253 (34), 219 (100), 199 (44), 190 (23), 180 (34), 172 (14), 156 (18), 144 (66), 116 (72). CHN Analysis: $\mathrm{C}_{21} \mathrm{H}_{26} \mathrm{FN}_{3} \mathrm{O}_{3}$ Cal: $\mathrm{C}$ $65.10 \%$, H 6.76\%, N 10.85\%, Found: C 65.13\%, H $6.78 \%, \mathrm{~N} 10.84 \%$.

General Procedure for Synthesis of Compounds (7-14) Methyl-1-Ethyl-6-Fluoro-1, 4-Dihydro-4Oxo-7-(Substituted Piperazin-1-Yl) Quinoline-3Carboxylate (7a-14a): A solution of methyl-7chloro- 1- ethyl- 6- fluoro-1, 4-dihydro- 4 oxoquinoline-3-carboxylate ( $1 \mathrm{~g}, 3.53 \mathrm{~m} \mathrm{~mol})$ added in acetonitrile $(10 \mathrm{ml})$, DIPEA $(0.5 \mathrm{ml})$ and substituted piperazine $(3.53 \mathrm{~m}$ mole) was added and shacked at boiling temperature for $24 \mathrm{~h}$. and kept an eye by TLC. The reaction mixture was quenched with water, juiced with ethyl acetate, dried over sodium sulphate, intense under reduced pressure to obtain basic product and it was purified by column chromatography using silica gel (100200 mesh) to get methyl-1-ethyl-6-fluoro-1, 4dihydro - 4 - oxo - 7 - (substituted piperazin-1-yl) quinoline-3-carboxylate as off white solid.

Comp. 7a: Yield (72\%, 0.84 g). MP- $190-192{ }^{\circ} \mathrm{C}$.

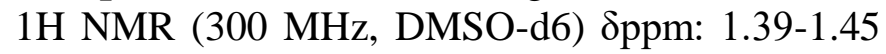
$\left(\mathrm{t}, 3 \mathrm{H}, \mathrm{CH}_{3}\right), 2.88-2.90\left(\mathrm{~s}, 4 \mathrm{H}\right.$, piperazine $\left.2 \mathrm{CH}_{2}\right)$, 3.21-3.24 (s, $4 \mathrm{H}$, piperazine $\left.2 \mathrm{CH}_{2}\right), 3.73(\mathrm{~s}, 3 \mathrm{H}$, $\left.\mathrm{OCH}_{3}\right), 3.80$ (m, 1H, NH), 4.55-4.62 (q, 2H, $\mathrm{CH}_{2}$ ),
7.12-7.15 (d, 1H, Ar H), 7.86-7.90 (d, 1H, Ar H), 8.94 (s, 1H, NC=C-H). IR (KBr) cm $\mathrm{cm}^{-1}: 3450(\mathrm{~N}-\mathrm{H}$ st), $2910\left(\mathrm{CH}\right.$ st, Alkyl), $1710\left(\mathrm{C}=\mathrm{O}, \mathrm{COOCH}_{3}\right)$, $1685(\mathrm{C}=\mathrm{O}$, quinoline $), 1592,1610(\mathrm{C}=\mathrm{C} \mathrm{Ar}), 1412$ (C-N st), 1372 (C-N-C=), 1260 (C-O-C).

Comp. 8a: Yield: (76\%, 0.92 g). MP- $158-160{ }^{\circ} \mathrm{C}$.

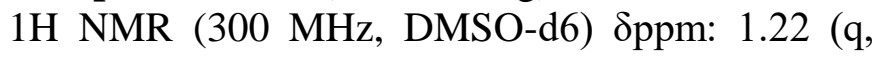
$1 \mathrm{H}$, near to $\left.\mathrm{CH}_{3}\right), 1.39-1.45\left(\mathrm{t}, 3 \mathrm{H}, \mathrm{CH}_{3}\right), 1.48$ (d, $\left.3 \mathrm{H}, \mathrm{CH}_{3}\right), 2.88-2.90\left(\mathrm{~s}, 4 \mathrm{H}\right.$, piperazine $\left.\mathrm{CH}_{2}\right), 3.21$ $3.20\left(\mathrm{~s}, 2 \mathrm{H}\right.$, piperazine $\left.\mathrm{CH}_{2}\right), 3.73\left(\mathrm{~s}, 3 \mathrm{H}, \mathrm{OCH}_{3}\right)$, $3.80(\mathrm{~m}, 1 \mathrm{H}, \mathrm{NH}), 4.55-4.62\left(\mathrm{q}, 2 \mathrm{H}, \mathrm{CH}_{2}\right), 7.12-$ 7.15 (d, 1H, Ar H), 7.86-7.90 (d, 1H, Ar H), 8.94 (s, 1H, C=C-H). IR $(\mathrm{KBr}) \mathrm{cm}^{-1}: 1592,1610(\mathrm{C}=\mathrm{C}$ Ar), 1412 (C-N st), 3450 (NH st), 1372 (C-N-C=), $2910\left(\mathrm{CH}\right.$ st, alkyl), $1710\left(\mathrm{C}=\mathrm{O}, \mathrm{COOCH}_{3}\right), 1680$ $(\mathrm{C}=\mathrm{O}$, quinoline $)$.

Comp. 9a: Yield: (78\%, 0.99 g). MP- $196-198^{\circ} \mathrm{C}$.

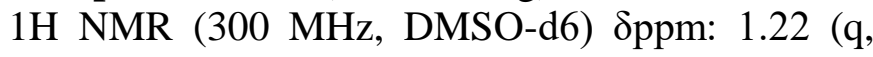
$2 \mathrm{H}$, near to $\left.\mathrm{CH}_{3}\right), 1.36-1.40\left(\mathrm{t}, 3 \mathrm{H}, \mathrm{CH}_{3}\right), 1.44(\mathrm{~d}$, $\left.6 \mathrm{H}, 2 \mathrm{CH}_{3}\right), 2.88-2.90\left(\mathrm{~s}, 4 \mathrm{H}\right.$, piperazine $\left.\mathrm{CH}_{2}\right), 3.75$ $\left(\mathrm{s}, 3 \mathrm{H}, \mathrm{OCH}_{3}\right), 3.80(\mathrm{~m}, 1 \mathrm{H}, \mathrm{NH}), 4.55-4.65(\mathrm{q}, 2 \mathrm{H}$, $\left.\mathrm{CH}_{2}\right), 7.12-7.15(\mathrm{~d}, 1 \mathrm{H}, \mathrm{Ar} \mathrm{H}), 7.86-7.90$ (d, 1H, Ar $\mathrm{H}), 8.94(\mathrm{~s}, 1 \mathrm{H}, \mathrm{C}=\mathrm{C}-\mathrm{H})$. IR $(\mathrm{KBr}) \mathrm{cm}^{-1}: 3450(\mathrm{~N}-$ H st), 2910 (C-H st, alkyl), $1710\left(\mathrm{C}=\mathrm{O}, \mathrm{COOCH}_{3}\right)$, $1680(\mathrm{C}=\mathrm{O}$, quinoline $), 1590,1610(\mathrm{C}=\mathrm{C} \mathrm{Ar}), 1414$ (C-N st), 1374 (C-N-C=), 1266 (C-O-C).

Comp. 10a: Yield: (70\%, 0.85 g). MP- 164-166 ${ }^{\circ} \mathrm{C}$. 1H NMR (300 MHz, DMSO-d6) Sppm: 1.35$1.45\left(\mathrm{t}, 3 \mathrm{H}, \mathrm{CH}_{3}\right), 2.88-2.90(\mathrm{~s}, 4 \mathrm{H}$, piperazine $\left.2 \mathrm{CH}_{2}\right), 3.10-3.40\left(\mathrm{~s}, 4 \mathrm{H}\right.$, piperazine $\left.2 \mathrm{CH}_{2}\right), 3.75(\mathrm{~s}$, $\left.3 \mathrm{H}, \mathrm{OCH}_{3}\right), 3.80$ (s, 3H, N-CH $), 4.55-4.65(\mathrm{q}, 2 \mathrm{H}$, $\left.\mathrm{CH}_{2}\right), 7.12-7.15$ (d, $\left.1 \mathrm{H}, \mathrm{Ar} \mathrm{H}\right), 7.86-7.90(\mathrm{~d}, 1 \mathrm{H}, \mathrm{Ar}$ $\mathrm{H}), 8.94$ (s, 1H, C=C-H). IR (KBr) cm ${ }^{-1}$ : 1590, 1610 (C=CAr), 1414 (C-N st), 1374 (C-N-C=), 2910 (C-H st, alkyl), 1710 (C=O, COOCH3), 1680 $(\mathrm{C}=\mathrm{O}$, quinoline), 1264 (C-O-C).

Comp. 11a: Yield (68\%, 0.89 g). MP- $160-162{ }^{\circ} \mathrm{C}$.

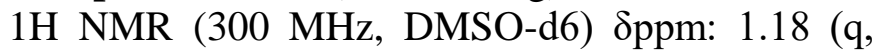
$1 \mathrm{H}$, piperazine $\mathrm{H}), 1.20-1.25(\mathrm{t}, 3 \mathrm{H}$, quinoline $\left.\mathrm{CH}_{3}\right), 1.35-1.38\left(\mathrm{t}, 3 \mathrm{H}\right.$, piperazine $\left.\mathrm{CH}_{3}\right), 1.40(\mathrm{~d}$, $3 \mathrm{H}, \mathrm{CH}_{3}$ ), 3.98 (q, 2H, quinoline $\mathrm{CH}_{2}$ ), 1.55-1.65 (q, 2H, piperazine $\mathrm{CH}_{2}$ ), 3.10 (br-s, $4 \mathrm{H}$, piperazine $\mathrm{CH}_{2}$ ), 3.40 (br-s, $2 \mathrm{H}$, piperazine $\mathrm{CH}_{2}$ ), 3.74 (s, $3 \mathrm{H}$, $\left.\mathrm{OCH}_{3}\right), 7.60-7.62$ (d, 1H, Ar H), 7.88-7.98 (d, 1H, $\operatorname{Ar} \mathrm{H}), 8.68$ (s, 1H, N-C=C-H). IR $(\mathrm{KBr}) \mathrm{cm}^{-1}$ : 2930 (C-H Ar), $1710\left(\mathrm{C}=\mathrm{O}, \mathrm{COOCH}_{3}\right), 1675(\mathrm{C}=\mathrm{O}$ quinoline), 1564, 1492 (C=C Ar), 1459 (C-N st), 1382 (C-N-C=), 1264 (C-O-C). 
Comp. 12a: Yield $\left(66 \%, 0.90\right.$ g). MP- $178-180{ }^{\circ} \mathrm{C}$.

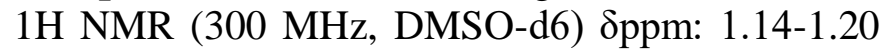
(t, $3 \mathrm{H}$, quinoline $\left.\mathrm{CH}_{3}\right), 1.25(\mathrm{q}, 2 \mathrm{H}$, piperazine $\mathrm{H})$, 1.35-1.40 (t, 3H, piperazine $\left.\mathrm{CH}_{3}\right), 3.96(\mathrm{q}, 2 \mathrm{H}$, $\left.\mathrm{CH}_{2}\right), 1.48\left(\mathrm{~d}, 6 \mathrm{H}, 2 \mathrm{CH}_{3}\right), 1.55-1.66(\mathrm{q}, 2 \mathrm{H}$, piperazine $\mathrm{CH}_{2}$ ), 2.90 (br-s, $4 \mathrm{H}$, piperazine $\mathrm{CH}_{2}$ ), $3.76\left(\mathrm{~s}, 3 \mathrm{H}, \mathrm{OCH}_{3}\right), 7.50-7.62(\mathrm{~d}, 1 \mathrm{H}, \mathrm{Ar} \mathrm{H}), 7.88-$ $7.90(\mathrm{~d}, 1 \mathrm{H}, \operatorname{Ar} \mathrm{H}), 8.64$ (s, 1H, N-C=C-H). IR $(\mathrm{KBr}) \quad \mathrm{cm}^{-1}: 2930 \quad(\mathrm{C}-\mathrm{H} \quad \mathrm{Ar}), 1710 \quad(\mathrm{C}=\mathrm{O}$, $\left.\mathrm{COOCH}_{3}\right), 1680$ (C=O quinoline), 1565, 1490 $(\mathrm{C}=\mathrm{C}$ Ar), 1456 (C-N st), 1380 (C-N-C=), 1268 (C$\mathrm{O}-\mathrm{C})$.

Comp. 13a: Yield: (62\%, 1.87 g). MP-252-254 ${ }^{\circ} \mathrm{C}$.

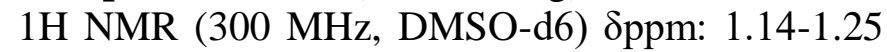
(t, $3 \mathrm{H}, \mathrm{CH}_{3}$ ), 3.40 (br-s, $4 \mathrm{H}$, piperazine $2 \mathrm{CH}_{2}$ ), 3.60 (br-s, $4 \mathrm{H}$, piperazine $2 \mathrm{CH}_{2}$ ), 4.10 (q, $2 \mathrm{H}, \mathrm{CH}_{2}$ ), $3.90\left(\mathrm{~s}, 3 \mathrm{H}, \mathrm{OCH}_{3}\right), 7.42-7.62(\mathrm{~m}, 5 \mathrm{H}), 7.90-7.97$ $(\mathrm{d}, 1 \mathrm{H}), 8.68(\mathrm{~s}, 1 \mathrm{H}, \mathrm{N}-\mathrm{C}=\mathrm{C}-\mathrm{H})$. IR $(\mathrm{KBr}) \mathrm{cm}^{-1}$ : 2922 (C-H Ar), 1710 (C=O, COOCH3), 1685 $(\mathrm{C}=\mathrm{O}$, quinoline $), 1564,1469(\mathrm{C}=\mathrm{C}$ Ar), 1380 (C$\mathrm{N} \mathrm{st}$ ), 1260 (C-O-C).

Comp. 14a: Yield: $\left(68 \%, 1.90\right.$ g). MP-248-250 ${ }^{\circ} \mathrm{C}$. 1H NMR (300 MHz, $\left.\mathrm{CDCl}_{3}\right) \delta p p m: 1.14-1.25(\mathrm{t}$, $3 \mathrm{H}, \mathrm{CH}_{3}$ ), 3.40 (br-s, $4 \mathrm{H}$, piperazine $2 \mathrm{CH}_{2}$ ), 3.60 (br-s, $4 \mathrm{H}$, piperazine $\left.2 \mathrm{CH}_{2}\right), 3.80\left(\mathrm{~s}, 3 \mathrm{H}, \mathrm{Ph} \mathrm{OCH}_{3}\right.$ ), 3.90 (s, 3H, ester $\left.\mathrm{OCH}_{3}\right), 4.10$ (q, $\left.2 \mathrm{H}, \mathrm{CH}_{2}\right), 7.42-$ $7.62(\mathrm{~m}, 5 \mathrm{H}), 7.90-7.97(\mathrm{~d}, 1 \mathrm{H}), 8.68(\mathrm{~s}, 1 \mathrm{H}, \mathrm{N}-$ $\mathrm{C}=\mathrm{C}-\mathrm{H})$. IR $(\mathrm{KBr}) \mathrm{cm}^{-1}$ : $2922(\mathrm{C}-\mathrm{H} \mathrm{Ar}), 1710$ $\left(\mathrm{C}=\mathrm{O}, \mathrm{COOCH}_{3}\right), 1682(\mathrm{C}=\mathrm{O}$, quinoline $), 1564$, $1470(\mathrm{C}=\mathrm{C}$ Ar), 1380 (C-N st), 1265 (C-O-C).

\section{1-Ethyl -6-Fluoro-1, 4-Dihydro-4-Oxo-7-} (Substituted Piperazin-1-Yl) Quinoline-3Carboxylic Acid (7-14): A solution of methyl-1ethyl-6-fluoro-1, 4-dihydro-4-oxo - 7 - (substituted piperazin-1-yl) quinoline-3-carboxylate $(2.1 \mathrm{~m}$ mol) in methanol $(10 \mathrm{ml})$, water $(4.2 \mathrm{ml})$, LiOH. $\mathrm{H}_{2} \mathrm{O}(0.34 \mathrm{ml}, 8.10 \mathrm{~m} \mathrm{~mol})$ was added, shacked at room temperature for $6 \mathrm{~h}$. TLC showed completion of starting material. The reaction mixture was quenched with water, washed with ethyl acetate. The aqueous layer was nullifying with $1 \mathrm{~N} \mathrm{HCl}$, juiced with ethyl acetate and dried over sodium sulphate, concentrated under reduced pressure.

The obtained basic product was refined by silica gel column chromatography using 30\% ethyl acetate in hexane as an eluent to get 1-ethyl-6- fluoro-1, 4-dihydro-4-oxo-7-(substituted piperazin1-yl) quinoline-3-carboxylic acid as white solid.

Comp. 7: Yield (65\%, 0.43 g). MP- $168-170{ }^{\circ} \mathrm{C}$.

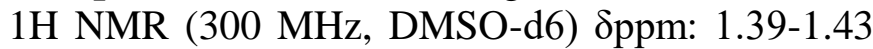
$\left(\mathrm{t}, 3 \mathrm{H}, \mathrm{CH}_{3}\right), 2.87-2.90\left(\mathrm{~s}, 4 \mathrm{H}\right.$, piperazine $\left.2 \mathrm{CH}_{2}\right)$, 3.21-3.24 (s, $4 \mathrm{H}$, piperazine $\left.2 \mathrm{CH}_{2}\right), 4.55-4.62(\mathrm{q}$, $\left.2 \mathrm{H}, \mathrm{CH}_{2}\right), 7.12-7.15(\mathrm{~d}, 1 \mathrm{H}, \mathrm{Ar} \mathrm{H}), 7.86-7.90$ (d, $1 \mathrm{H}, \operatorname{Ar} \mathrm{H}), 8.94(\mathrm{~s}, 1 \mathrm{H}, \mathrm{C}=\mathrm{C}-\mathrm{H}), 15.20(\mathrm{~s}, 1 \mathrm{H}$, $\mathrm{COOH})$. IR (KBr) cm $\mathrm{cm}^{-1}: 3640(\mathrm{OH}, \mathrm{COOH}), 3450$ (N-H st), 2910 (C-H st, alkyl), 1710 (C=O, $\mathrm{COOH}), 1685(\mathrm{C}=\mathrm{O}$, quinoline $), 1590,1610$ (C=C $\mathrm{Ar}$ ), 1410 (C-N st), $1370(\mathrm{C}-\mathrm{N}-\mathrm{C}=)$. MS: m/z (rel int\%) 319 (24) [M+], 296 (18), 290 (24), 278 (45), 255 (48, 219 (56), 199 (100), 182 (65), 156 (68), 152 (73), 130 (70), 113 (78), 108(48). CHN Analysis: $\mathrm{C}_{16} \mathrm{H}_{18} \mathrm{FN}_{3} \mathrm{O}_{3}$ Cal: $\mathrm{C}$ 60.12\%, H 5.63\%, N $13.15 \%$, Found: C 60.16\%, H 5.62\%, N 13.16\%,

Comp. 8: Yield: $\left(60 \%, 0.40\right.$ g). MP- $146-148{ }^{\circ} \mathrm{C}$.

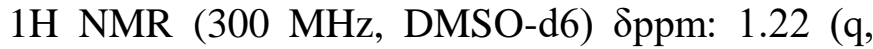
$1 \mathrm{H}$, near to $\left.\mathrm{CH}_{3}\right), 1.40\left(\mathrm{~d}, 3 \mathrm{H}, \mathrm{CH}_{3}\right), 1.42-1.46(\mathrm{t}$, $\left.3 \mathrm{H}, \mathrm{CH}_{3}\right), 2.88-2.90\left(\mathrm{~s}, 4 \mathrm{H}\right.$, piperazine $\left.\mathrm{CH}_{2}\right), 3.80$ (m, 1H, NH), 3.21-3.20 (s, 2H, piperazine $\mathrm{CH}_{2}$ ), 4.55-4.65 (q, 2H, $\left.\mathrm{CH}_{2}\right), 7.12-7.15(\mathrm{~d}, 1 \mathrm{H}, \mathrm{Ar} \mathrm{H})$, 7.86-7.90 (d, 1H, Ar H), $8.94(\mathrm{~s}, 1 \mathrm{H}, \mathrm{C}=\mathrm{C}-\mathrm{H})$, 15.20 (s, 1H, COOH). IR (KBr) cm $\mathrm{cm}^{-1}: 3550$ (COOH), 3450 (N-H st), 2910 (C-H st, alkyl), 1710 $(\mathrm{C}=\mathrm{O}, \mathrm{COOH}), 1680(\mathrm{C}=\mathrm{O}$, quinoline $), 1592,1610$ $(\mathrm{C}=\mathrm{C}$ Ar), 1412 (C-N st), 1372(C-N-C=). MS: m/z (rel int\%) 333 (18) [M+], 296 (25), 288 (16), 274 (41), 255 (55), 219 (58), 199 (100), 176 (65), 156 (66), 154 (70), 132 (75), 116 (88), 106 (58). CHN Analysis: $\mathrm{C}_{17} \mathrm{H}_{20} \mathrm{FN}_{3} \mathrm{O}_{3}$ Cal: $\mathrm{C} 61.25 \%, \mathrm{H} 6.05 \%$, N 12.61\%, Found: C 60.30\%, H 6.10\%, N $12.68 \%$.

Comp. 9: Yield: (58\%, 0.38 g). MP- $180-182{ }^{\circ} \mathrm{C}$.

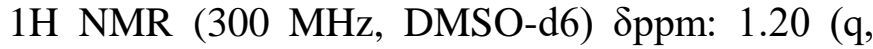
$2 \mathrm{H}$, near to $\left.\mathrm{CH}_{3}\right), 1.28\left(\mathrm{~d}, 6 \mathrm{H}, 2 \mathrm{CH}_{3}\right), 1.42-1.45$ (t, $\left.3 \mathrm{H}, \mathrm{CH}_{3}\right), 2.88-2.90$ (s, $4 \mathrm{H}$, piperazine $\left.\mathrm{CH} 2\right), 3.80$ (m, 1H, NH), 4.58-4.65 (q, 2H, $\left.\mathrm{CH}_{2}\right), 7.12-7.15$ (d, $1 \mathrm{H}, \operatorname{Ar} \mathrm{H}), 7.86-7.90(\mathrm{~d}, 1 \mathrm{H}, \mathrm{Ar} H), 8.94(\mathrm{~s}, 1 \mathrm{H}$, $\mathrm{C}=\mathrm{C}-\mathrm{H}), 15.20(\mathrm{~s}, 1 \mathrm{H}, \mathrm{COOH}) . \mathrm{IR}(\mathrm{KBr}) \mathrm{cm}^{-1}$ : 3550 (COOH), 3450 (N-H st), 2910 (C-H st, alkyl), $1710(\mathrm{C}=\mathrm{O}, \mathrm{COOH}), 1680(\mathrm{C}=\mathrm{O}$, quinoline $), 1592$, 1610 (C=C Ar), 1412 (C-N st), 1372 (C-N-C=). MS: m/z (relint\%) 347 (22) [M+], 312 (25), 292 (19), 272 (38), 258 (52), 214 (52), 198 (100), 178 (68), 151 (60), 142 (78), 132 (74), 114 (82), 104(38). CHN Analysis: $\mathrm{C}_{18} \mathrm{H}_{22} \mathrm{FN}_{3} \mathrm{O}_{3}$ Cal: C 
$62.23 \%, \mathrm{H} 6.38 \%, \mathrm{~N} 12.10 \%$, Found: C $62.30 \%, \mathrm{H}$ $6.45 \%, \mathrm{~N} 12.18 \%$.

Comp. 10: Yield: $(62 \%, 0.41 \mathrm{~g})$. MP-151-153 ${ }^{\circ} \mathrm{C}$.

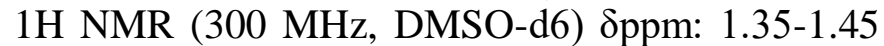
(t, 3H, $\left.\mathrm{CH}_{3}\right), 3.82$ (s, 3H, N-CH 3$), 2.88-2.95$ (s, 4H, piperazine $\mathrm{CH} 2), 3.21-3.24(\mathrm{~s}, 2 \mathrm{H}$, piperazine $\mathrm{CH}_{2}$ ), 4.55-4.65 (q, 2H, CH$)_{2}, 7.12-7.15$ (d, 1H, $\mathrm{Ar}$ H), 7.86-7.90 (d, 1H, Ar H), $8.94(\mathrm{~s}, 1 \mathrm{H}, \mathrm{C}=\mathrm{C}-\mathrm{H})$, $15.20(\mathrm{~s}, 1 \mathrm{H}, \mathrm{COOH})$. IR $(\mathrm{KBr}) \mathrm{cm}^{-1}: 3550(\mathrm{OH}$ st, $\mathrm{COOH}), 2910(\mathrm{CH}$ st, alkyl), $1710(\mathrm{C}=\mathrm{O}, \mathrm{COOH})$, $1680 \quad(\mathrm{C}=\mathrm{O}, \quad$ quinoline $), \quad 1590, \quad 1610 \quad(\mathrm{C}=\mathrm{C}$ aromatic), 1420 (C-N st), $1374(\mathrm{C}-\mathrm{N}-\mathrm{C}=)$. MS: m/z (rel int\%) 333 (18) [M+], 318 (20), 292 (18), 266 (32), 258 (50), 222 (100), 200 (60), 178 (68), 158 (45), 152 (74), 136 (80), 116 (87), 98 (56). CHN Analysis: $\mathrm{C}_{17} \mathrm{H}_{20} \mathrm{FN}_{3} \mathrm{O}_{3}$ Cal: $\mathrm{C}$ 61.25\%, H 6.05\%, N $12.61 \%$, Found: C 61.30\%, H 6.10\%, N $12.70 \%$.

Comp. 11: Yield: (64\%, 0.42 g). MP- $146-148{ }^{\circ} \mathrm{C}$.

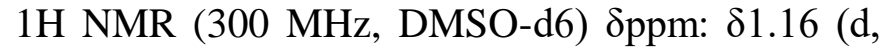
$\left.3 \mathrm{H}, \mathrm{CH}_{3}\right), 1.18-1.26\left(\mathrm{t}, 3 \mathrm{H}, \mathrm{CH}_{3}\right), 1.31(\mathrm{q}, 1 \mathrm{H}$, piperazine $\mathrm{H}$ ), 1.45-1.48 (t, $\left.3 \mathrm{H}, \mathrm{CH}_{3}\right), 3.10$ (br-s, $4 \mathrm{H}$, piperazine $\mathrm{CH}_{2}$ ), 3.40 (br-s, $2 \mathrm{H}$, piperazine $\mathrm{CH} 2$ ), 3.90 (q, 2H, $\mathrm{CH}_{2}$ ), 4.55-4.65 (q, 2H, $\mathrm{CH}_{2}$ ), 7.60-7.65 (d, 1H, Ar H), 7.84-7.98 (d, 1H, Ar H), $8.66(\mathrm{~s}, 1 \mathrm{H}, \mathrm{N}-\mathrm{C}=\mathrm{C}-\mathrm{H}), 15.20(\mathrm{~s}, 1 \mathrm{H}, \mathrm{COOH})$. IR $(\mathrm{KBr}) \mathrm{cm}-1: 3550$ (OH st, $\mathrm{COOH}), 2930$ (C-H Ar), $1710(\mathrm{C}=\mathrm{O}, \mathrm{COOH}), 1620(\mathrm{C}=\mathrm{O}$ quinoline $), 1564$, 1494 ( $\mathrm{C}=\mathrm{C}$ Ar), 1460 (C-N st), 1384 (C-N-C=). MS: $\mathrm{m} / \mathrm{z}$ (relint\%) 361 (28) [M+], 352 (18), 322 (20), 296 (31), 272 (38), 248 (100), 224 (51), 202 (58), 188 (40), 162 (70), 148 (76), 126 (78), 102 (45), 92(40). CHN Analysis: $\mathrm{C}_{19} \mathrm{H}_{24} \mathrm{FN}_{3} \mathrm{O}_{3}$ Cal: $\mathrm{C}$ $63.14 \%$, H 6.69\%, N 11.63\%, Found: C 63.20\%, H $6.75 \%, \mathrm{~N} 11.70 \%$.

Comp. 12: Yield $\left(68 \%, 0.45\right.$ g). MP-160-162 ${ }^{\circ} \mathrm{C}$.

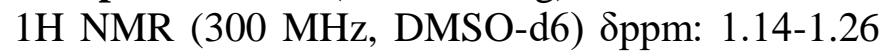
(t, 3H, CH3), 1.26 (q, 2H, piperazine H), 1.35-1.40 (t, 3H, $\left.\mathrm{CH}_{3}\right), 1.48\left(\mathrm{~d}, 6 \mathrm{H}, 2 \mathrm{CH}_{3}\right), 2.90(\mathrm{br}-\mathrm{s}, 4 \mathrm{H}$, piperazine $\mathrm{CH}_{2}$ ), 3.90 (q, $\left.2 \mathrm{H}, \mathrm{CH}_{2}\right), 4.58-4.66$ (q, $\left.2 \mathrm{H}, \mathrm{CH}_{2}\right), 7.50-7.62$ (d, 1H, Ar H), 7.88-7.90(d, $1 \mathrm{H}$, Ar H), $8.64(\mathrm{~s}, 1 \mathrm{H}, \mathrm{N}-\mathrm{C}=\mathrm{C}-\mathrm{H}), 15.22(\mathrm{~s}, 1 \mathrm{H}$, $\mathrm{COOH})$. IR (KBr) cm $\mathrm{cm}^{-1}: 3550(\mathrm{O}-\mathrm{H}$ st, $\mathrm{COOH})$, 2930 (C-H Ar), 1710 (C=O, COOH), $1630(\mathrm{C}=\mathrm{O}$ quinoline), 1565, 1490, (C=C Ar), 1456(C-N st), $1380(\mathrm{C}-\mathrm{N}-\mathrm{C}=)$. MS: m/z (relint\%) $375(16)[\mathrm{M}+]$, 366 (21), 346 (23), 320 (29), 292 (30), 282 (38), 272 (56), 254 (62), 199 (100), 158 (82), 136 (72), 118 (76), 102 (40), 90 (32). CHN Analy: $\mathrm{C}_{20} \mathrm{H}_{26} \mathrm{~F}$
$\mathrm{N}_{3} \mathrm{O}_{3}$ Cal: C 63.98\%, H 6.98\%, N 11.19\%, Found: C 64.04\%, H 7.02\%, N $11.25 \%$

Comp. 13: Yield (72\%, 0.48 g). MP-225-227 ${ }^{\circ} \mathrm{C}$.

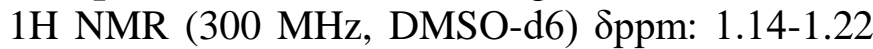
(t, $3 \mathrm{H}, \mathrm{CH}_{3}$ ), 3.40 (br-s, $4 \mathrm{H}$, piperazine $2 \mathrm{CH}_{2}$ ), 3.60 (br-s, 4H, piperazine $\left.2 \mathrm{CH}_{2}\right), 3.90\left(\mathrm{q}, 2 \mathrm{H}, \mathrm{CH}_{2}\right.$ ), 7.42-7.62 (m, 4H, Ar H), 7.90-7.97 (d, 1H, ArH), 8.68 (s, s, 1H, N-C=C-H), 15.20 (s, 1H, COOH). IR (KBr) cm $\mathrm{cm}^{-1}: 3550$ (O-H st, COOH), 2922 (C-H Ar), $1720(\mathrm{C}=\mathrm{O}, \mathrm{COOH}), 1710(\mathrm{C}=\mathrm{O}, \mathrm{CCON}), 1628$ $(\mathrm{C}=\mathrm{O}$, quinoline $), 1564,1469(\mathrm{C}=\mathrm{C} \mathrm{Ar}), 1380(\mathrm{C}-\mathrm{N}$ st). MS: m/z (relint\%) 492(12) [M+], 480(11), 462 (14), 446 (21), 412 (19), 395 (22), 378 (26), 332 (20), 284 (31), 272 (26), 240 (41), 214 (43), 196 (100), 165 (30), 146 (32), 120 (46), 98 (46). CHN Analy: $\mathrm{C}_{23} \mathrm{H}_{20} \mathrm{C}_{12} \mathrm{FN}_{3} \mathrm{O}_{4}$ Cal: C $56.11 \%$, H 4.09\%, N 08.53\%, Found: C 56.20\%, H 4.16\%, N 08.62\%

Comp. 14: Yield $\left(76 \%, 0.50\right.$ g). MP- $228-230{ }^{\circ} \mathrm{C}$.

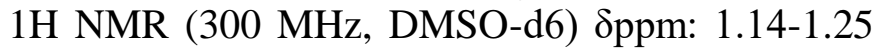
(t, $3 \mathrm{H}, \mathrm{CH}_{3}$ ), 3.40 (br-s, $4 \mathrm{H}$, piperazine $2 \mathrm{CH}_{2}$ ), 3.60 (br-s, 4H, piperazine $\left.2 \mathrm{CH}_{2}\right), 3.90\left(\mathrm{q}, 2 \mathrm{H}, \mathrm{CH}_{2}\right)$, 7.42-7.62 (m, 5H, Ar H), 7.90-7.97 (d, 1H, Ar H), $8.68(\mathrm{~s}, \mathrm{~s}, 1 \mathrm{H}, \mathrm{N}-\mathrm{C}=\mathrm{C}-\mathrm{H}), 3.82\left(\mathrm{~s}, 3 \mathrm{H}, \mathrm{OCH}_{3}\right)$. $15.20(\mathrm{~s}, 1 \mathrm{H}, \mathrm{COOH})$. IR $(\mathrm{KBr}) \mathrm{cm}^{-1}: 3550(\mathrm{O}-\mathrm{H}$ st, $\mathrm{COOH}), 2922(\mathrm{C}-\mathrm{H}$ Ar), $1720(\mathrm{C}=\mathrm{O}, \mathrm{COOH})$, $1710(\mathrm{C}=\mathrm{O}, \mathrm{CCON}), 1628(\mathrm{C}=\mathrm{O}$, quinoline $), 1564$, $1470(\mathrm{C}=\mathrm{C}$ Ar), $1380(\mathrm{C}-\mathrm{N}$ st $) . \mathrm{MS}: \mathrm{m} / \mathrm{z}$ (relint\%) 453(11) $[\mathrm{M}+], 442$ (12), 418 (16), 402 (16), 391 (18), 370 (24), 345 (21), 308 (24), 284 (33), 272 (21), 242 (44), 214 (32), 196 (100), 166 (30), 148 (36), 120 (48), 94 (66). CHN Analy: $\mathrm{C}_{24} \mathrm{H}_{24} \mathrm{FN}_{3} \mathrm{O} 5$ Cal: C $63.57 \%$, H $5.33 \%$, N $09.27 \%$, Found: C $63.65 \%$, H $5.40 \%$, N $09.35 \%$

\section{Biological Assays:}

Cells and Viruses: Assay was executed at the Molecular Immuno pharmacology Laboratory, Kunming Zoology Institute, Chinese Academy of Science. Reagents made available in laboratory as HEPES (N-2 (2-Hydroxyothyl) piperazine-N'-(2ethanesufonic acid) and MTT (3, (4, 5 dimethyl thiazol-2-yl) -2, 5-diphenyl tetrazolium bromide) and DMF (N, N'- Dimethyl formamine).

Penicillin, Streptomycin sulfate, Glutamine were procured from Sigma; 2-ME (2-Mercapto ethanol) was obtained from Bio-Rad. RPMI-1640 and fetal bovine serum (FBS) were obtained from Gibco. C8166 cells and HIV-1IIIB were kindly contributed by the Medical Research Council, AIDS Regent 
Project. The cells were sustained at $37{ }^{\circ} \mathrm{C}$ in $5 \%$ $\mathrm{CO}_{2}$ in RPMI-1640 medium supplemented with $10 \%$ heat-inactivating FBS (Gibco). HIV-1IIIB was ready from the floating of $\mathrm{H} 9 / \mathrm{HIV}-1 \mathrm{IIIB}$ cells. The $50 \%$ HIV-1 tissue culture infectious dose (TCID50) in C8166 cells was firm and designed by Reed and Muench method ${ }^{14-15}$. Virus stock was maintained in a small fraction at $-76{ }^{\circ} \mathrm{C}$. The virus titer stock was $1.0 \times 108$ TCID50 per ml.

\section{In-vitro Assays:}

Inhibition of Syncytia Formation: The syncytia formation assay was used to measure the inhibition effect of samples on acute HIV-1 infection. In the attendance or nonexistence of dissimilar sample concentrations, 4, 1104, 8166 cells were contaminated with HIV-1 at a multiplicity of 0.04 infections (MOI) and grown for $72 \mathrm{~h}$ in 96-well plates at $37{ }^{\circ} \mathrm{C}$ in $5 \% \mathrm{CO}_{2}$. As an affirmative control, NVP was used. Cytopathic effect (CPE) was measured at $72 \mathrm{~h}$ post-infection by together with the number of syncytia (multinucleated giant cell) under an inverted microscope (100) in each well of 96 well plates. The blockage percentage of syncytia formation was calculated by the percentage of syncytia number in the sample being treated compared to the percentage in the control being infected. An effective concentration of 50 percent $\left(\mathrm{EC}_{50}\right)$ was calculated ${ }^{16}$.

Cytotoxicity: MTT colorimetric assay checked the cell toxicity of compounds on C8166 cells.15 Momentarily, $100 \mu \mathrm{l}$ of 4 possibly 105 cells were plated in 96 well plates, $100 \mu \mathrm{l}$ of diverse compound concentrations were added and constructed at $37{ }^{\circ} \mathrm{C}$ in a socked atmosphere of $5 \%$ $\mathrm{CO}_{2}$ for $72 \mathrm{~h}$. Discard $100 \mu \mathrm{l}$ airy liquid, added and nurtured MTT reagent for $4 \mathrm{~h}$, added $50 \%$ DMF, $15 \%$ SDS of $100 \mu \mathrm{l}$. After the total ending of the formazan, a Bio Tek ELx 800 ELISA reader analyzed the plates at $570 \mathrm{~nm} / 630 \mathrm{~nm}$. The attentiveness of cytotoxicity $\left(\mathrm{CC}_{50}\right)$ was calculated at $50 \%$.

HIV-1 Integrase Inhibition Assay: The enzymatic examinations were done with small changes as per the above method ${ }^{17}$. We used an enzyme-linked immunosorbent assay (ELISA) adapted from Hwang et $a l .{ }^{14}$ to decide the susceptibility of the HIV-1 IN enzyme to different compounds. The assay adopted was an oligonucleotide substrate for which one oligonucleotide (5'-ACTGCTAGAGA TTTTCCACACTGACTAAAAGGGTC-3') is designated with biotin at the 3 ' end and the other oligonucleotide (5'-GACCCTTTTAGTCAGTGTG GAAAATCTCTAGCAGT-3') is labeled with digoxigenin at the $5^{\prime}$ end. The strand transfer examination, a dissected oligonucleotide substrate (the second oligonucleotide lacks GT [underlined] at the 3 ' end) was accounted. The IN enzyme was thinned in $750 \mathrm{mM} \mathrm{NaCl}, 10 \mathrm{mM}$ Tris ( $\mathrm{pH}$ 7.6), $10 \%$ glycerol, and $1 \mathrm{mM} \beta$-mercaptoethanol. To carry out the reaction, $4 \mu \mathrm{l}$ of thinned IN (corresponding to a concentration of $1.6 \mu \mathrm{M}$ ) and 4 $\mu 1$ of annealed oligonucleotides $(7 \mathrm{nM})$ were mixed in a ending reaction volume of $40 \mu \mathrm{l}$ containing 10 $\mathrm{mM} \mathrm{MgCl}_{2}, 5 \mathrm{mM}$ dithiothreitol, $20 \mathrm{mM}$ HEPES $(\mathrm{pH} 7.5), 5 \%$ polyethylene glycol and $15 \%$ dimethyl sulfoxide. As such, the ending concentration of IN in this assay was $160 \mathrm{nM}$. The reaction was passed out for $1 \mathrm{~h}$ at $37{ }^{\circ} \mathrm{C}$. Reaction products were altered with $30 \mathrm{mM} \mathrm{NaOH}$ and realized by ELISA on avidin-coated plates.

For shaping the effect of compounds on the 3 'processing activity a usual cleavage assay with the finding of products by altering gel electrophoresis was done as mentioned earlier1617. In a few words, $0.2 \mathrm{pmol}$ of the radioactively labeled oligonucleotide substrate (INT1, 32P-5' TGTGGAAA ATCTCTAGCAGT 3'; INT2, 5'ACTGCTAGAGA TTTTCCACA 3') and 10 nmol IN in a final volume of $10 \mu 1$ was constructed for $1 \mathrm{~h}$ at $37 \mathrm{o} \mathrm{C}$. The last reaction mixture enclosed $20 \mathrm{mM}$ HEPES $\mathrm{pH}$ 7.5), $5 \mathrm{mM}$ dithiothreitol (DTT), $10 \mathrm{mM} \mathrm{MgCl}_{2}, 0.5 \%$ (v/v) polyethylene glycol 8000, 15\% DMSO, IN was thinned earlier in $750 \mathrm{mM} \mathrm{NaCl}, 10 \mathrm{mM}$ Tris $(\mathrm{pH}$ 7.6), $10 \%$ glycerol and $1 \mathrm{mM} \beta$ mercaptoethanol. The reactions were blocked by the accumulation of formamide loading buffer (95\% formamide, $0.1 \%$ xylene cyanol, $0.1 \%$ xylene cyanol, $0.1 \%$ bromophenol blue and $0.1 \%$ sodium dodecyl sulfate). The specimen was full on a $15 \%$ denaturating polyacrylamide/ureum gel. The level of 3' processing or DNA strand transfer depended on measuring the own amounts of -2 bands or strand transfer products comparative to the intensity of the sum radioactivity present in the lane. The statistics were resolute using the OptiQuant Acquisition and Analysis software (Perkin Elmer Corporate, Fremont, CA). 
Drug Susceptibility Assay: The MTT examination was done to check the suppression effect of antiviral drugs on the HIV-1induced CPE in MT-4 cell culture ${ }^{18}$. This examination is placed on the drop of the yellow-colored 3-(4, 5-dimethylthiazol2 yl)-2, 5-diphenyltetrazolium bromide (MTT) by mitochondrial dehydrogenase of metabolically lively cells to a blue formazan derivative, which can be considered spectro-photometrically. The $50 \%$ cell culture infective dose of the HIV strains was firm by titration of the virus stock using MT-4 cells.

The compound weakness assays, MT-4 cells were infected with 100 to $30050 \%$ cell culture infective doses of the HIV strains in the presence of fivefold successive strength of the antiviral drugs. The attention of the compound achieving 50\% protection against the CPE of HIV, which is defined as the $50 \%$ effective concentration $\left(\mathrm{IC}_{50}\right)$, was firm. The amount of the compound killing $50 \%$ of the MT-4 cells, which is defined as the $50 \%$ cytotoxic concentration $\left(\mathrm{CC}_{50}\right)$, was determined as well.

\section{Molecular Modelling:}

Docking: For ligand docking studies in the HIV-1 Integrase pouch, the molecular docking tool, GLIDE was used. The structure of HIV-1 Integrase crystal was received from the database of proteins, PDB ID: 1QS4. In two steps, preparation and refinement, the protein preparation was performed using the ' protein preparation wizard in Maestro 8.0. On co-crystallized ligand centering grids were generated. The ligands were prepared by the maestro build panel and ready by the Ligprep 2.2 module, which uses the OPLS 2005 force field to create the low energy ligands conformer. The low energy conformation of the ligands was selected and docked with standard precision (SP) docking mode into the grid generated from protein structure.

\section{RESULTS AND DISCUSSION:}

Design of the Analogues: The series of compounds to be considered for study was carried out using the virtual screening protocol ${ }^{18-20}$. Considering the pharmacophoric requirements and the Elvitegravir standard compound, the database of Zinc was explored. The compounds screened in silico were then tested for the five fold Lipinsky rule to assess drug-likeness, which becomes a crucial means for drug innovation. SciFinder has been checked for the novelty of compounds in terms of HIV-1 Integrase inhibitory activity. These virtually screened hits have been synthesized and evaluated for their inhibitory potential along with their derivatives. It was found that for HIV-I Integrase activity, diketoquinolines were not much explored. We, therefore, studied the motif of diketoquinoline for inhibition of Integrase. Two series of the compound were synthesized as diketoquinoline analogs.

Chemistry: The target diketoquinolines 1-14 were prepared from the commercially procured intermediates such as methyl-7-chloro-1-cyclopropyl-6fluoro-1, 4-dihydro-4-oxoquinoline-3-carboxylate, methyl- 7- chloro-1-ethyl-6-fluoro-1, 4-dihydro-4oxo Quinoline-3-carboxylate and 1-ethyl-6-fluoro1, 4-dihydro-4-oxo-7-(piperazin-1-yl) quinoline-3carboxylate ${ }^{21-26}$. The fabricated way is marked in Scheme 1.

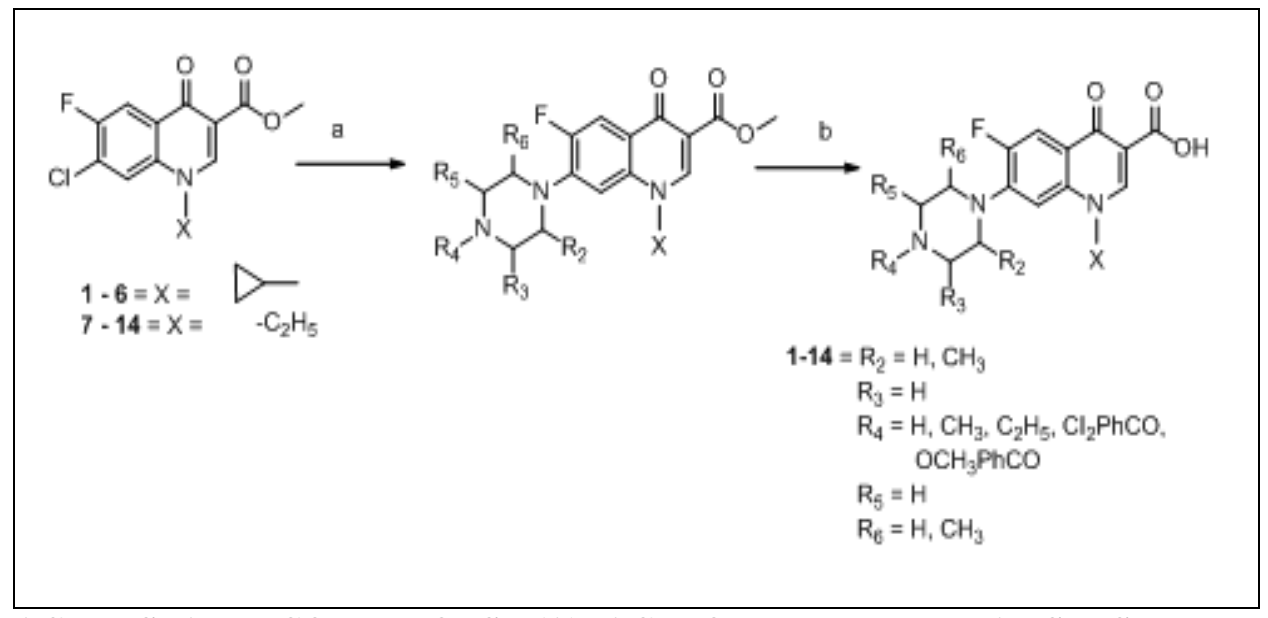

SCHEME 1: REAGENTS AND CONDITIONS: (A) ACETONITRILE, DIPEA, SUBSTITUTED PIPERAZINE, REFLUXED 24 H, ETHYL ACETATE; (B) METHANOL, WATER, LIOH.H 2 , STIRRED 6 H 
All the carboxylate compounds were reacted with substituted piperazine, benzoic acid, via substitution of chlorine by piperazine nitrogen in presence of DIPEA (N, N-di isopropyl ethyl amine) to produce the intermediate compound in excellent yield. In the same way, ring extension at the nitrogen of piperazine was carried out by substituted benzoic acid. The ester linkage in intermediate compound $\left(\mathrm{COOCH}_{3}\right)$ is hydrolyzed to a carboxylic acid $(\mathrm{COOH})$ in an alkaline medium in the attendance of melted lithium hydroxide to yield the target compounds ${ }^{1-14}$.
The confirmation of removal of $\mathrm{C}-\mathrm{Cl}$ and attachment of substituted compounds and conversion of carboxylate to carboxylic acid was verified by spectral analysis.

Biological Activity: Diketoquinoline derivatives 114 were tested in vitro for their cytotoxicity assay and inhibition of syncytia formation using MTT and $\mathrm{CPE}$ method 8-9 $\mathrm{CC}_{50}$ and $\mathrm{EC}_{50}$ reading was obtained from replica experiments in $\mu \mathrm{M}$ and selectivity index was also planned by dose-reaction loop Table 1.

TABLE 1: CYTOTOXICITY (MTT) AND INHIBITION OF SYNCYTIUM FORMATION (CPE) ACTIVITIES OF COMPOUNDS 1-14

\begin{tabular}{|c|c|c|c|c|}
\hline Comp. no. & Method & $\mathrm{CC}_{50}(\mu \mathrm{M})$ & $\mathrm{EC}_{50}(\mu \mathrm{M})$ & Therapeutic index (TI) \\
\hline \multirow[t]{2}{*}{1} & MTT & 148 & - & 242 \\
\hline & CPE & - & 0.61 & \\
\hline \multirow[t]{2}{*}{2} & MTT & 130 & - & 260 \\
\hline & CPE & - & 0.50 & \\
\hline \multirow[t]{2}{*}{3} & MTT & 128 & - & 266 \\
\hline & CPE & - & 0.48 & \\
\hline \multirow[t]{2}{*}{4} & MTT & 128 & - & 278 \\
\hline & CPE & - & 0.46 & \\
\hline \multirow[t]{2}{*}{5} & MTT & 140 & - & 341 \\
\hline & CPE & - & 0.41 & \\
\hline \multirow[t]{2}{*}{6} & MTT & 150 & - & 394 \\
\hline & CPE & - & 0.38 & \\
\hline \multirow[t]{2}{*}{7} & MTT & 155 & - & 430 \\
\hline & CPE & - & 0.36 & \\
\hline \multirow[t]{2}{*}{8} & MTT & 162 & - & 395 \\
\hline & CPE & - & 0.41 & \\
\hline \multirow[t]{2}{*}{9} & MTT & 172 & - & 554 \\
\hline & CPE & - & 0.31 & \\
\hline \multirow[t]{2}{*}{10} & MTT & 180 & - & 545 \\
\hline & CPE & - & 0.33 & \\
\hline \multirow[t]{2}{*}{11} & MTT & 188 & - & 587 \\
\hline & CPE & - & 0.32 & \\
\hline \multirow[t]{2}{*}{12} & MTT & 198 & - & \\
\hline & CPE & - & 0.31 & 638 \\
\hline \multirow[t]{2}{*}{13} & MTT & $>200$ & - & 714 \\
\hline & CPE & - & 0.28 & \\
\hline \multirow[t]{2}{*}{14} & MTT & $>200$ & - & 823 \\
\hline & CPE & --- & 0.25 & \\
\hline \multirow[t]{2}{*}{ Nevirapine } & MTT & $>200$ & -- & 12500 \\
\hline & $\mathrm{CPE}$ & - & 0.016 & \\
\hline
\end{tabular}

As per the previous study for Diketoquinoline derivative series 27 acidic derivatives were more potent for a maximum sensation against integrase. The alternate of a hydrophobic ring from C6 of Elvitegravir by Flourine and $\mathrm{C} 7$ by substituted piperazine moiety did not direct to major upgrading in the HIV-1 integrase blocking effect. Substitution at quinoline $\mathrm{N}$ by ethyl and piperazine $\mathrm{N}$ by hydrophobic phenyl carbonyl group resulted in compounds 13 and 14 which showed $\mathrm{IC}_{50}$ of 0.13 and $0.12 \mu \mathrm{M}$ against integrase enzyme respectively.
This suggests that substitution of quinoline $\mathrm{N}$ by alkyl and a hydrophobic moiety at C6 (Elvitegravir) or at $\mathrm{C} 7$ piperazine $\mathrm{N}$ affect the ability of the inhibitors to bind with integrase enzyme. Other substitution does not make any significant interaction with HIV-1 integrase enzyme Table 2.

Molecular Docking: The putative binding mechanism of the synthesized compounds was investigated using molecular docking studies. The 
docking studies of the designed and synthesized molecules were performed using Schrodinger Suite. The molecular docking device, GLIDE was utilized for ligand docking observation into the HIV-1 integrase pouch ${ }^{28-30}$. The crystal formation of HIV1 integrase was taken from the protein data bank, PDB ID: 1QS4.

The preparation of protein was done by device 'protein preparation wizard' in Maestro 8.0 in two ladders such as groundwork and improvement. Grids were generated centering on the cocrystallized ligand. The ligands were established by using the maestro build panel and arranged by the Ligprep 2.2 module that generates the small power conformer of ligands using the OPLS 2005 force field. The small power arrangement of the ligands was chosen and docked into the lattice prepared from protein arrangement using standard precision (SP) docking manner.

As per the literature, the active site comprises of Thr 66, Lys 156, Lys 159 and DDE motif (Asp 64, Asp 116 and Glu 152). The docked poses revealed the interaction of a few ligands with desired amino acids. The standard drug Elvitegravir showed a docking score of -8.93 and displayed interactions with lys 156, Asn 155, Lys 159 and Thr 66 Fig. A. When Elvitegravir and Nevirapine were docked in the same active site, they displayed comparable docking scores and interaction patterns. Raltegravir revealed hydrogen bonding with Asp 116 while Nevirapine showed hydrogen bonding with Asp 64. The synthesized compounds which displayed fair integrase inhibition were also docked in order to ascertain the interactions and were compared with a standard pose. Compound 14 exhibited maximum potency with $\mathrm{EC}_{50}$ of $0.12 \mathrm{mM}$ and $0.25 \mathrm{mM}$ in enzyme inhibition and cell line assay respectively. Its methoxy group was involved in hydrogen bonding with Gln 146. It also showed interactions with Lys 156, Lys 159 and Asp 116 Fig. B. Other compounds were also evaluated for ligand-protein interaction and compared with the standard drug.

The unsubstituted piperazine in compound 1 favored the hydrogen bonding interaction with Asp 116 through the $-\mathrm{NH}$ of piperazine ring system while the carbonyl group on the quinoline system displayed hydrogen bond with Thr 66. Positioning two methyl substituents on the piperazine system (compound 3) helped a molecule align in such a manner that the carboxyl group extended its interactions with Lys 156 and Lys 159.

Its $\mathrm{EC}_{50}$ value was $0.22 \mathrm{mM}$ and $0.48 \mathrm{mM}$ in enzyme inhibition and cell line assay respectively. The series with compounds ${ }^{7-14}$ having N-ethyl substituted quinoline also displayed a similar type of interaction pattern with improved activity than the previous series. The docking analysis also shows that though the compounds favored interactions with desired amino acids but none of the compounds could show interactions with the important $\mathrm{Mg}^{2+}$ ions i.e. $\mathrm{Mg} 1001$ and $\mathrm{Mg} 1002$.

Compound 8 displayed strong hydrogen bonding interactions through its carboxyl group with Lys 156, Lys 159 and piperazine $\mathrm{NH}$ showed interaction with Glu 92. Its $\mathrm{EC}_{50}$ value was 0.16 $\mathrm{mM}$. A similar type of interaction pattern was revealed by other compounds of this series. Some additional interactions were observed with Asp 64 and Asp 116 in the compounds 9, 10, 11, 12, 13 and 14 . The activity profile was concomitant with the docking analysis. The docking analysis also revealed that the interaction pattern of the designed compounds was similar to the standard drug Elvitegravir. The docking scores are given in Table 2 and Fig. 2 represents the docked view.

TABLE 2: CORRELATION OF DOCKING SCORE AND CYTOTOXICITY AND ANTI-INTEGRASE ACTIVITIES OF SYNTHESIZED COMPOUNDS 1-14

\begin{tabular}{ccccc}
\hline Comp no. & $\begin{array}{c}\text { Docking } \\
\text { score }\end{array}$ & $\begin{array}{c}(\boldsymbol{\mu M}) \\
\mathbf{E C}_{\mathbf{5 0}}\end{array}$ & $\begin{array}{c}(\boldsymbol{\mu M}) \\
\mathbf{C C}_{\mathbf{5 0}}\end{array}$ & SI \\
\hline 1 & -6.536 & 0.31 & $>200$ & 645 \\
2 & -7.877 & 0.25 & $>200$ & 800 \\
3 & -7.235 & 0.22 & $>200$ & 909 \\
4 & -6.523 & 0.21 & 196 & 933 \\
5 & -7.984 & 0.18 & 195 & 1083 \\
6 & -7.890 & 0.17 & 191 & 1123 \\
7 & -8.683 & 0.17 & $>200$ & 1176 \\
8 & -7.802 & 0.16 & 192 & 1200 \\
9 & -7.635 & 0.15 & 198 & 1320 \\
10 & -7.771 & 0.14 & 178 & 1271 \\
11 & -8.113 & 0.14 & 190 & 1357 \\
12 & -7.985 & 0.13 & 196 & 1507 \\
13 & -7.465 & 0.13 & $>200$ & 1538 \\
14 & -8.138 & 0.12 & $>200$ & 1666 \\
Raltegravir & -4.291 & 0.0111 & ND & ND \\
\hline
\end{tabular}

Not Determined (ND), Selective Index (SI)

Note: When data indicate $>200$ for $\mathrm{EC}_{50}$ and $\mathrm{CC}_{50}$ it means that the compounds were neither active not toxic at $200 \mu \mathrm{M}$ which is the highest concentration we can test to stay in the DMSO tolerance levels. When data indicates a number lower than 200 for $\mathrm{EC}_{50}$ and then the same number with the equality sign in the $\mathrm{CC}_{50}$ it means that we observed toxicity at this concentration. 


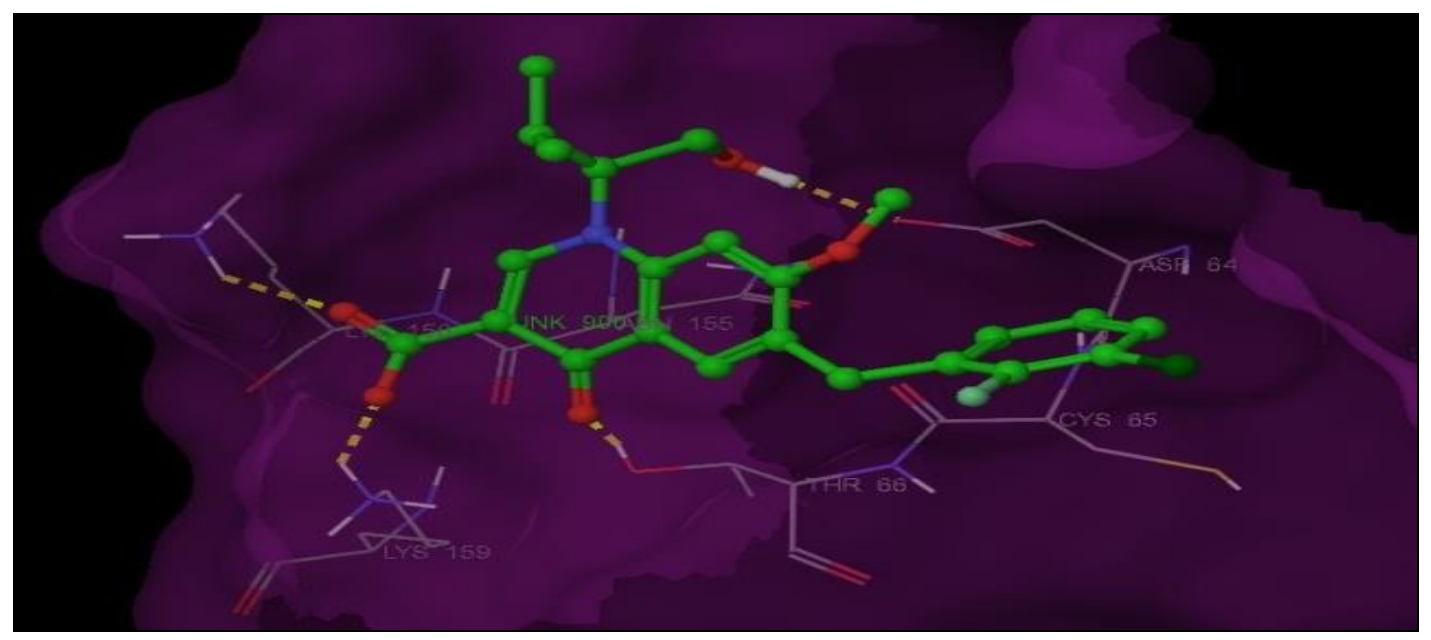

A. DOCKED POSE VIEW OF ELVITEGRAVIR IN THE ACTIVE SITE OF HIV-1 IN (PDB ID: 1QS4). ACTIVE SITE NUCLEIC ACID RESIDUES WERE REPRESENTED AS STICKS. REST OF THE NUCLEIC ACID RESIDUES ARE SUPPRESSED FOR CLARIFICATION PURPOSE. HYDROGEN BOND INTERACTIONS ARE REPRESENTED BY DOTTED LINES

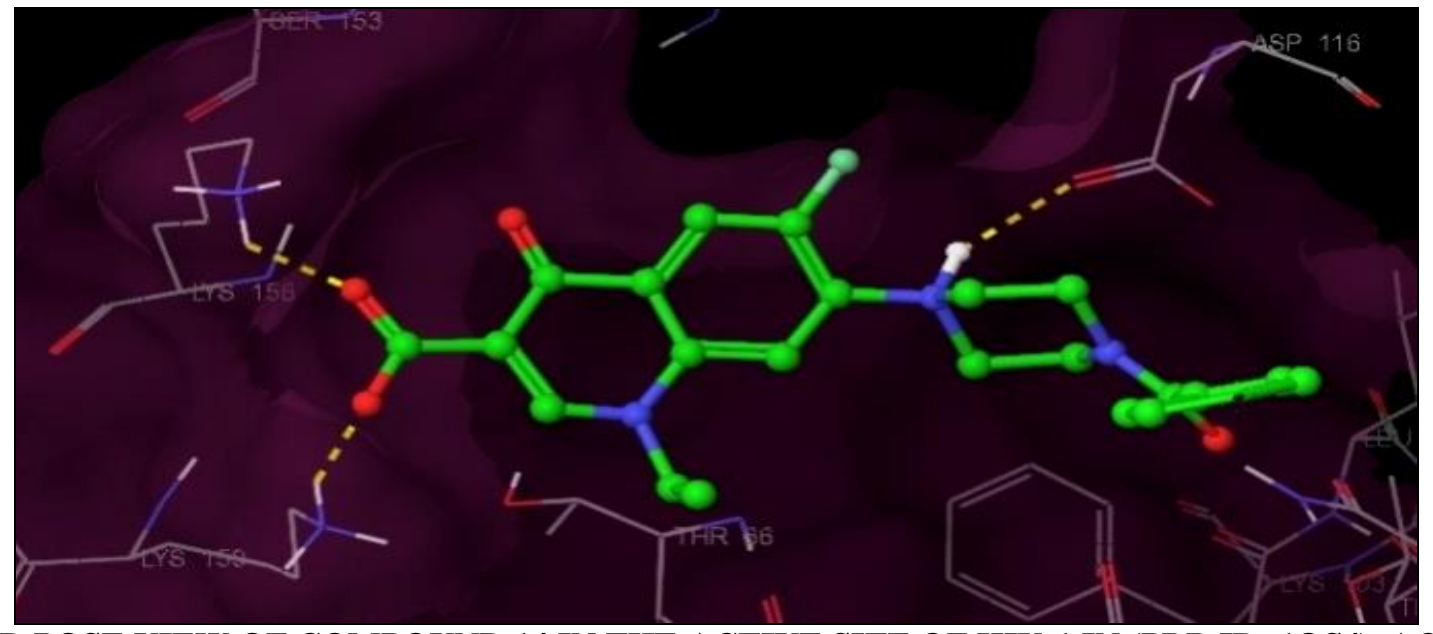

B. DOCKED POSE VIEW OF COMPOUND 14 IN THE ACTIVE SITE OF HIV-1 IN (PDB ID: 1QS4). ACTIVE SITE NUCLEIC ACID RESIDUES WERE REPRESENTED AS STICKS. REST OF THE NUCLEIC ACID RESIDUES ARE SUPPRESSED FOR CLARIFICATION PURPOSE. HYDROGEN BOND INTERACTIONS ARE REPRESENTED BY DOTTED LINES

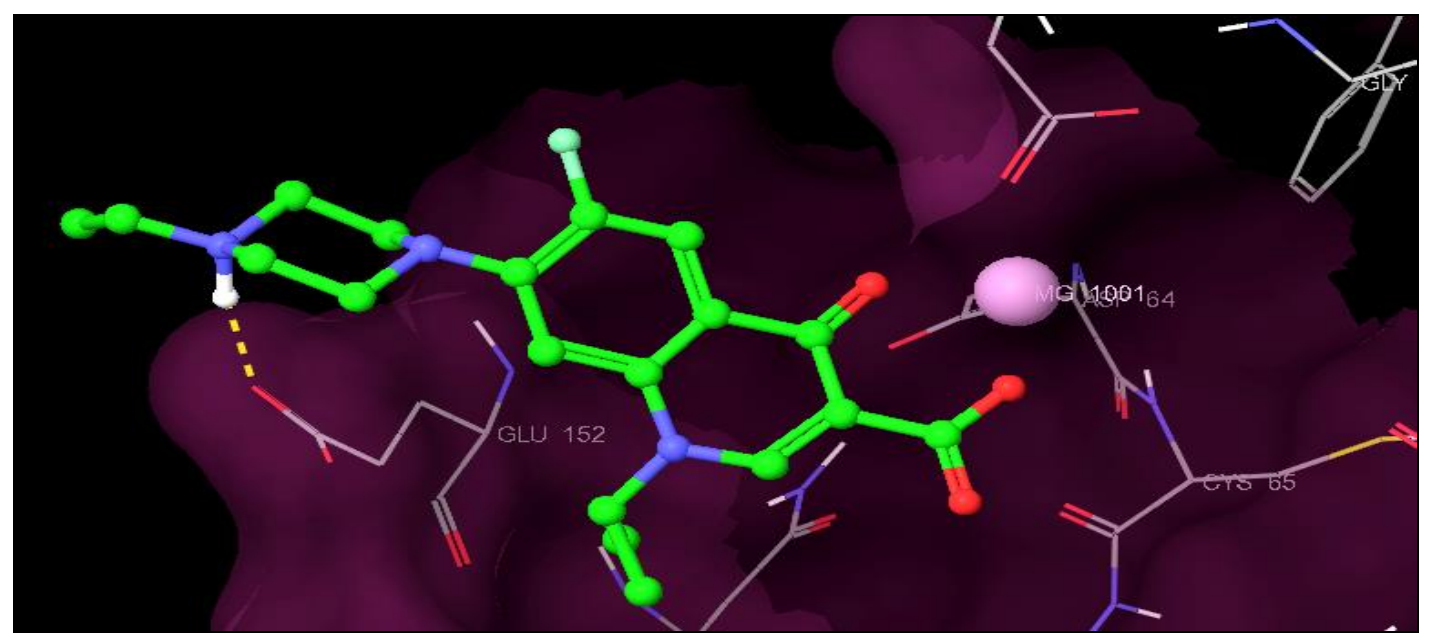

C. DOCKED POSE VIEW OF COMPOUND 3 IN THE ACTIVE SITE OF HIV-1 IN (PDB ID: 1QS4). ACTIVE SITE NUCLEIC ACID RESIDUES WERE REPRESENTED AS STICKS. REST OF THE NUCLEIC ACID RESIDUES ARE SUPPRESSED FOR CLARIFICATION PURPOSE. HYDROGEN BOND INTERACTIONS ARE REPRESENTED BY DOTTED LINES 
CONCLUSION: To make clear the precise binding mode of the upper-class diketoacid quinolonyl chain of compounds, fresh derivatives were prepared by altering the 6-position with Fluorine atom instead of Elvitegravir's replaced hydrophobic benzyl moiety and replacing piperazine, benzoic acid, 2-phenoxyacetic acid and benzene-1-sulfonyl chloride at 7-position substitution. For their enzymatic action, these compounds have been examined.

Replacing Elvitegravir C6 hydrophobic ring with F and $\mathrm{C} 7$ by piperazine group does not result in a major development in integrase blocking of HIV-1. Substitution at quinoline $\mathrm{N}$ by ethyl and piperazine $\mathrm{N}$ by hydrophobic phenyl carbonyl group 13, 14 showed $\mathrm{IC}_{50}$ value 0.13 and $0.12 \mu \mathrm{M}$ against integrase enzyme. This result suggests that substitution of quinoline $\mathrm{N}$ by alkyl and a hydrophobic moiety at C6 (Elvitegravir) or at C7 piperazine $\mathrm{N}$ by phenyl carbonyl affect the ability of the inhibitors to bind with integrase enzyme. Other substitution does not make any significant interaction with HIV-1 integrase enzyme. Hence further lead optimization studies will be required to find more potent HIV-1 In inhibitors.

ACKNOWLEDGEMENT: We are very grateful to the Director and Laboratory of Molecular Immunopharmacology, Kunming Zoology Institute, Chinese Academy of Science 32 Jiaochang Donglu Kunming, Yunnan 650223, China for carrying out the compound and support anti - HIV activity. Thanks to the University of Pune for spectral analysis and thanks to Professor Dr. H. N. Patel, R. C. Patel College of Pharmacy, Shirpur for providing kind support for molecular modeling study.

CONFLICTS OF INTEREST: Authors articulate that they neither have any disagreement of interest.

\section{REFERENCES:}

1. Masoudnia E: Public perceptions about HIV/AIDS and discriminatory attitudes toward people living with acquired immunodeficiency syndrome in Iran. SAHARA J Soc Asp HIV/AIDS Res Alliance 2015; 12(1): 116-22.

2. Vancampfort D, Mugisha J, Richards J, De Hert M, Probst M and Stubbs B: Physical activity correlates in people living with HIV/AIDS: a systematic review of 45 studies. Disability and Rehabilitation 2018; 40(14): 1618-29.

3. Choi E, Reddy J, Mallareddy D and Kolluru S: Recent advances in the discovery of small-molecule inhibitors of HIV-1 integrase. Future Sci OA 2018; 4(9): FSO338.
4. Kale M and Puthoor A: Molecular modeling in search of newer anti-HIV agents: A review. Der Pharmacia Sinica 2016; 7(30): 10-22.

5. Giuliana R, Romina P, Valerio I, Ilaria T, Martina M, Matteo P, Roberto D, Alessandro D, Beatrice M, Caterina F, Gianpiero F, Antonella D, Gustavo C P and Paolo B: Synthesis of potential HIV integrase inhibitors inspired by natural polyphenol structures. Natural Product Research 2018; 32(16): 1893-01.

6. Thakur DS, Behera CK, Patidar A and Kumar P: Anti-hiv agents: a step towards future. International Journal of Pharmaceutical Sciences Review and Research 2010; 3(2): 314-22.

7. Mehellou Y and Clercq ED: Twenty-six years of anti-hiv drug discovery: where do we stand and where do we go? J Med Chem 2010; 53: 521-38.

8. Tabarrini O, Massari S and Cecchetti V: 6desfluoroquinolones as HIV-1 Tat-mediated transcription inhibitors. Future Medicinal Chemistry 2010; 2(7): 116180 .

9. Clavel F and Hance AJ: HIV drug resistance. J Med 2004; 350: 1023-35.

10. David LH: Review of synthetic routes and final forms of integrase inhibitors dolutegravir, cabotegravir and bictegravir. Org Process Res Dev 2019; 23(5): 716-29.

11. Crum NF, Riffenburgh RH, Wegner S, Agan BK, Tasker, SA and Spooner KM: Comparisons of causes of death and mortality rates among HIV-infected persons: analysis of the pre-early and late HAART (highly active antiretroviral therapy) eras. J Acq Immu Defic Syndr 2006; 41: 194-200.

12. Dybul MF, Bartlett AS, Kaplan JG and Pau AK: Guidelines for using antiretroviral agents among HIVinfected adults and adolescents. Ann Intern Med 2002; 137: 381-33.

13. Engelman A and Cherepanov P: The structural biology of HIV-1: mechanistic and therapeutic insights. Nat Rev Microbiol 2012; 10(4): 279-90.

14. Hwang Y, Rhodes and Bushman F: Rapid microtiter assays for poxvirus topoisomerase, mammalian type IB topoisomerase and HIV-1 integrase: application to inhibitor isolation. Nucleic Acids Res 2000; 28: 4884-92.

15. Pauwels RJ, Balzarini M, Baba R, Snoeck D, Schols P, Herdewijn J, Desmyter E and De C: Rapid and automated tetrazolium-based colorimetric assay for the detection of anti-HIV compounds. J Virol Methods 1988; 20: 309-21.

16. Christ F, Busschots K, Hendrix J, Melissa, McNeely, Yves E and Debyser Z: Assays for evaluation of hiv-1 integrase enzymatic activity, DNA binding, and cofactor interaction. Nouri Neamati (ED) John Wiley \& Sons Inc 2011; 1(1): 1508.

17. Debyser Z, Cherepanov P, Pluymers W and De Clercq E: Assays for the evaluation of HIV-1 integrase inhibitors. In: "Methods in Molecular Biology", Nuclease Methods and Protocols. Humana Press, Totowa, 2001, 1(160): 1-525.

18. Hopkins AL, Ren J, Milton J, Hazen RJ and Chan JH: Design of non-nucleoside inhibitors of hiv-1 reverse transcriptase with improved drug resistance properties. J Med Chem 2004; 47: 5912-22.

19. Freeman GA, Andrews CW and III Hopkins AL: Design of Non-nucleoside inhibitors of hiv-1 reverse transcriptase with improved drug resistance properties. J Med Chem 2004; 47: 5923-36.

20. Sato M, Motomura T, Aramaki H, Matsuda T, Yamashita $\mathrm{M}$ and Ito $\mathrm{Y}$ : Novel HIV-1 integrase inhibitors derived from quinolone antibiotics. J Med Che 2006; 49: 1506-08.

21. Luo Z, Zeng C, Wang F, He H, Wang C, Du H and Hu L: Synthesis and biological activities of quinoline derivatives 
as HIV-1 integrase inhibitors, Chem. Res. Chinese Universities 2009; 25(6): 841-45.

22. Ahmed N, Keyur GB, Sabde S, Mitra D, Singh IP and Bhutani KK: Synthesis and anti-HIV activity of alkylated quinoline 2, 4-diols, Bioorganic \& Medicinal Chemistry 2010; 18: 2872-79.

23. Sriram D, Bal TR and Yogeeswari P: Design, synthesis and biological evaluation of novel non-nucleoside HIV-1 reverse transcriptase inhibitors with broad-spectrum chemotherapeutic properties. Bioorganic and Medicinal Chemistry 2004; 12: 5865-73.

24. Chen S, Chen R, He M, Pang R, Tan Z and Yang M: Design, synthesis, and biological evaluation of novel quinoline derivatives as HIV-1 Tat-TAR interaction inhibitors. Bioorganic and Medicinal Chemistry 2009; 17: 1948-56.

25. Sancineto L, Iraci N, Barreca ML, Massari S and Manfroni G: Exploiting the anti-HIV 6-desfluoroquinolones to design multiple ligands. Bioorganic and Medicinal Chemistry 2014; 22: 4658-66.

26. Benard C, Zouhiri F, Bayle MN and Danet M: Linkermodified quinoline derivatives targeting HIV-1 integrase: synthesis and biological activity. Bioorganic and Medicinal Chemistry Letters 2004; 14: 2473-76.
27. Santo RD, Costi R, Roux A and Artico M: 2006 Novel Bifunctional Quinolonyl Diketo Acid Derivatives as HIV1Integrase Inhibitors: Design, Synthesis, Biological Activities and Mechanism of Action. J Med Chem 2006; 49(6): 1939-45.

28. Billamboz M, Bailly F, Barreca ML, Luca LD, Franc J and Mouscadet O: Design, Synthesis, and Biological Evaluation of a Series of 2-Hydroxyisoquinoline-1, 3(2H, $4 \mathrm{H}$ )-diones as Dual Inhibitors of Human Immunodeficiency Virus Type 1 Integrase and the Reverse Transcriptase RNase H Domain. J Med Chem 2008; 51: 7717-30.

29. Billamboz M, Bailly F, Lion C and Calmels C: 2Hydroxyisoquinoline-1, 3(2H, $4 \mathrm{H})$-diones as inhibitors of HIV-1 integrase and reverse transcriptase RNase $\mathrm{H}$ domain: Influence of the alkylation of position 4. European Journal of Medicinal Chemistry 2011; 46: 53546.

30. Ghanei S, Eshghi H, Lari J and Saadatmandzadeh M: Synthesis and docking analysis of new 2-chloro-3-((2, 2dimethylhydrazono) methyl)quinoline derivatives as nonnucleoside human HIV-1 reverse transcriptase inhibitors. Journal of Chemical and Pharmaceutical Research 2015; 7(2): 428-33.

\section{How to cite this article:}

Deo KD, Singhvi IJ, Murugesan S, Vadnere GP and Patil AV: Design, synthesis and biological evaluation of novel quinoline analogues as HIV-1 integrase inhibitor. Int J Pharm Sci \& Res 2020; 11(3): 1210-23. doi: 10.13040/IJPSR.0975-8232.11(3).1210-23.

All @ 2013 are reserved by the International Journal of Pharmaceutical Sciences and Research. This Journal licensed under a Creative Commons Attribution-NonCommercial-ShareAlike 3.0 Unported License.

This article can be downloaded to Android OS based mobile. Scan QR Code using Code/Bar Scanner from your mobile. (Scanners are available on Google Playstore) 